\title{
Parameterizing edge modification problems above lower bounds
}

\author{
René van Bevern • Vincent Froese • \\ Christian Komusiewicz
}

October 13, 2018

\begin{abstract}
We study the parameterized complexity of a variant of the $F$-FREE EDITING problem: Given a graph $G$ and a natural number $k$, is it possible to modify at most $k$ edges in $G$ so that the resulting graph contains no induced subgraph isomorphic to $F$ ? In our variant, the input additionally contains a vertex-disjoint packing $\mathcal{H}$ of induced subgraphs of $G$, which provides a lower bound $h(\mathcal{H})$ on the number of edge modifications required to transform $G$ into an $F$-free graph. While earlier works used the number $k$ as parameter or structural parameters of the input graph $G$, we consider instead the parameter $\ell:=k-h(\mathcal{H})$, that is, the number of edge modifications above the lower bound $h(\mathcal{H})$. We develop a framework of generic data reduction rules to show fixed-parameter tractability with respect to $\ell$ for $K_{3}$-Free Editing, FeEdback Arc Set in Tournaments, and Cluster Editing when the packing $\mathcal{H}$ contains subgraphs with bounded solution size. For $K_{3}$-FreE Editing, we also prove NP-hardness in case of edge-disjoint packings of $K_{3} \mathrm{~s}$ and $\ell=0$, while for $K_{q}$-FreE Editing and $q \geq 6$, NP-hardness for $\ell=0$ even holds for vertex-disjoint packings of $K_{q}$ s. In addition, we provide NP-hardness results for $F$-Free Vertex Deletion, were the aim is to delete a minimum number of vertices to make the input graph $F$-free.
\end{abstract}

An extended abstract of this article appeared in Proceedings of the 11th International Computer Science Symposium in Russia, June 9-13, 2016, St. Petersburg, Russian Federation [6].

René van Bevern is supported by grant 16-31-60007 mol_a_dk of the Russian Foundation for Basic Research.

Christian Komusiewicz is supported by grant KO 3669/4-1 of Deutsche Forschungsgemeinschaft.

\section{René van Bevern}

Novosibirsk State University, Novosibirsk, Russian Federation, E-mail: rvb@nsu.ru

Sobolev Institute of Mathematics, Siberian Branch of the Russian Academy of Sciences, Novosibirsk,

Russian Federation

Vincent Froese

Technische Universität Berlin, Germany, E-mail: vincent.froese@tu-berlin.de

Christian Komusiewicz

Friedrich-Schiller-Universität Jena, Germany, E-mail: christian.komusiewicz@uni-jena.de 
Keywords. NP-hard problem, fixed-parameter algorithm, subgraph packing, kernelization, graph-based clustering, feedback arc set, cluster editing

\section{Introduction}

Graph modification problems are a core topic of algorithmic research [10, 34, 47]. Given a graph $G$, the aim is to transform $G$ by a minimum number of modifications (like vertex deletions, edge deletions, or edge insertions) into another graph $G^{\prime}$ fulfilling certain properties. Particularly well-studied are hereditary graph properties, which are closed under vertex deletions and are characterized by minimal forbidden induced subgraphs: a graph fulfills such a property if and only if it does not contain a graph $F$ from a property-specific family $\mathcal{F}$ of graphs as induced subgraph. All nontrivial vertex deletion problems and many edge modification and deletion problems for establishing hereditary graph properties are NP-complete $[1,3,33,34,47]$. One approach to cope with the NP-hardness of these problems are fixed-parameter algorithms that solve them in $f(k) \cdot n^{O(1)}$ time for some exponential function $f$ depending only on some desirably small parameter $k$. If the desired graph property has a finite forbidden induced subgraph characterization, then the corresponding vertex deletion, edge deletion, and edge modification problems are fixed-parameter tractable parameterized by the number of modifications $k$, that is, solvable in $f(k) \cdot n^{O(1)}$ time [10].

Parameterization above lower bounds. When combined with data reduction and pruning rules, search-tree based fixed-parameter algorithms for the parameter $k$ of allowed modifications can yield competitive problem solvers [26, 38]. Nevertheless, the number of modifications is often too large and smaller parameters are desirable.

A natural approach to obtain smaller parameters is "parameterization above guaranteed values" $[13,22,36,37]$. The idea is to use a lower bound $h$ on the solution size and to use $\ell:=k-h$ as parameter instead of $k$. This idea has been applied successfully to Vertex Cover, the problem of finding at most $k$ vertices such that their deletion removes all edges (that is, all $K_{2} \mathrm{~s}$ ) from $G$. Since the size of a smallest vertex cover is large in many input graphs, parameterizations above the lower bounds "size of a maximum matching $M$ in the input graph" and "optimum value $L$ of the LP relaxation of the standard ILP-formulation of VERTEX COVER" have been considered. After a series of improvements $[13,22,36,43]$, the current best running time is $3^{\ell} \cdot n^{O(1)}$, where $\ell:=k-(2 \cdot L-|M|)[22]$.

We extend this approach to edge modification problems, where the number $k$ of modifications tends to be even larger than for vertex deletion problems. For example, in the case of Cluster Editing, which asks to destroy induced paths on three vertices by edge modifications, the number of modifications is often larger than the number of vertices in the input graph [8]. Hence, parameterization above lower bounds seems natural and even more relevant for edge modification problems. Somewhat surprisingly, this approach has not been considered so far. We thus initiate research on parameterization above lower bounds in this context. As a starting point, we focus on edge modification problems for graph properties that are characterized by one small forbidden induced subgraph $F$ : 


\section{Problem 1.1 ( $F$-Free Editing)}

Input: A graph $G=(V, E)$ and a natural number $k$.

Question: Is there an $F$-free editing set $S \subseteq\left(\begin{array}{l}V \\ 2\end{array}\right)$ of size at most $k$ such that $G \triangle S:=$

$(V,(E \backslash S) \cup(S \backslash E))$ does not contain $F$ as induced subgraph?

In the context of a concrete variant of $F$-FREE EDITING, we refer to an $F$-free editing set as solution and call a solution optimal if it has minimum size.

Lower bounds from packings of bounded-cost induced subgraphs. Following the approach of parameterizing VERTEX COVER above the size of a maximum matching, we can parameterize $F$-FREE EDITING above a lower bound obtained from packings of induced subgraphs containing $F$.

Definition 1.2 A vertex-disjoint (or edge-disjoint) packing of induced subgraphs of a graph $G$ is a set $\mathcal{H}=\left\{H_{1}, \ldots, H_{z}\right\}$ such that each $H_{i}$ is an induced subgraph of $G$ and such that the vertex sets (or edge sets) of the $H_{i}$ are mutually disjoint.

While it is natural to consider packings of $F$-graphs to obtain a lower bound on the solution size, a packing of other graphs that contain $F$ as induced subgraph might yield better lower bounds and thus a smaller parameter above this lower bound. For example, a $K_{4}$ contains several triangles and two edge deletions are necessary to make it triangle-free. Thus, if a graph $G$ has a vertex-disjoint packing of $h_{3}$ triangles and $h_{4} K_{4}$ s, then at least $h_{3}+2 \cdot h_{4}$ edge deletions are necessary to make it trianglefree. ${ }^{1}$ Moreover, when allowing arbitrary graphs for the packing, the lower bounds provided by vertex-disjoint packings can be better than the lower bounds provided by edge-disjoint packings of $F$. A disjoint union of $h K_{4}$ s, for example, has $h$ edge-disjoint triangles but also $h$ vertex-disjoint $K_{4} \mathrm{~s}$. Hence, the lower bound provided by packing vertex-disjoint $K_{4} \mathrm{~s}$ is twice as large as the one provided by packing edge-disjoint triangles in this graph.

Motivated by this benefit of vertex-disjoint packings of arbitrary graphs, we mainly consider lower bounds obtained from vertex-disjoint packings, which we assume to receive as input. Thus, we arrive at the following problem, where $\tau(G)$ denotes the minimum size of an $F$-free editing set for a graph $G$ :

Problem 1.3 ( $F$-free Editing with Cost- $t$ Packing)

Input: A graph $G=(V, E)$, a vertex-disjoint packing $\mathcal{H}$ of induced subgraphs of $G$ such that $1 \leq \tau(H) \leq t$ for each $H \in \mathcal{H}$, and a natural number $k$.

Question: Is there an $F$-free editing set $S \subseteq\left(\begin{array}{l}V \\ 2\end{array}\right)$ of size at most $k$ such that $G \triangle S:=$

$(V,(E \backslash S) \cup(S \backslash E))$ does not contain $F$ as induced subgraph?

The special case of $F$-Free Editing with Cost- $t$ Packing where only $F$-graphs are allowed in the packing is called $F$-FreE Editing with $F$-PAcKING.

From the packing $\mathcal{H}$, we obtain the lower bound $h(\mathcal{H}):=\sum_{H \in \mathcal{H}} \tau(H)$ on the size of an $F$-free editing set, which allows us to use the excess $\ell:=k-h(\mathcal{H})$ over this lower bound as parameter, as illustrated in Figure 1.1. Since $F$ is a fixed graph, we

\footnotetext{
1 Bounds of this type are exploited, for example, in so-called cutting planes, which are used in speeding up the running time of ILP solvers.
} 

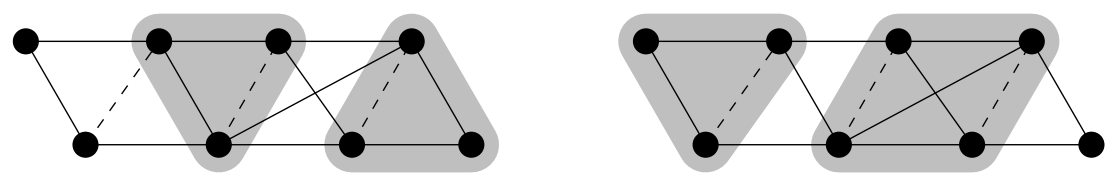

Fig. 1.1 An instance of Triangle Deletion. The packing graphs have gray background. Left: A vertexdisjoint packing of two triangles giving $\ell=1$. Right: A vertex-disjoint packing of a triangle and a $K_{4}$ giving $\ell=0$. The solution consists of the three dashed edges.

can compute the bound $h(\mathcal{H})$ in $f(t) \cdot|G|^{O(1)}$ time using the generic algorithm [10] mentioned in the introduction for each $H \in \mathcal{H}$. In the same time we can also verify whether the cost- $t$ property is fulfilled.

Packings of forbidden induced subgraphs have been used in implementations of fixed-parameter algorithms to prune the corresponding search trees tremendously [26]. By showing fixed-parameter algorithms for parameters above these lower bounds, we hope to explain the fact that these packings help in obtaining fast algorithms.

Our Results. We first state the negative results since they justify the focus on concrete problems and, to a certain extent, also the focus on parameterizing edge modification problems above lower bounds obtained from vertex-disjoint packings. We show that $K_{6}$-Free Editing with $K_{6}$-PAcking is NP-hard for $\ell=0$. This proves, in particular, that a general fixed-parameter tractability result as it is known for the parameter $k$ [10] cannot be expected. Moreover, we show that, if $F$ is a triangle and $\mathcal{H}$ is an edgedisjoint packing of $h$ triangles in a graph $G$, then it is NP-hard to decide whether $G$ has a triangle deletion set of size $h$ (that is, $\ell=0$ ). Thus, parameterization by $\ell$ is hopeless for this packing lower bound. We also consider vertex deletion problems. For these we show that extending the parameterization "above maximum matching" for Vertex Cover to $d$-Hitting Set in a natural way leads to intractable problems. This is achieved by showing that, for all $q \geq 3, P_{q}$-FreE Vertex Deletion with $P_{q}$-Packing is NP-hard even if $\ell=0$.

Our positive results are fixed-parameter algorithms and problem kernels (a notion for provably effective polynomial-time data reduction, see Section 2 for a formal definition) for three variants of $F$-Free Editing with Cost- $t$ Packing. Namely, these are the variants in which $F$ is a triangle (that is, a $K_{3}$ ) or a path on three vertices (that is, a $P_{3}$ ). The first case is known as Triangle Deletion, the second one as Cluster EDITING. We also consider the case in which the input is a tournament graph and $F$ is a directed cycle on three vertices. This is known as Feedback Arc Set in Tournaments. Using a general approach described in Section 3, we obtain fixed-parameter algorithms for these variants of $F$-Free Editing with Cost- $t$ Packing parameterized by $t$ and $\ell$. This implies fixed-parameter tractability for $F$-FREE EDITING WITH $F$-PACKING parameterized by $\ell$. Specifically, we obtain the following positive results:

(i) For Triangle Deletion, we show an $O\left((2 t+3)^{\ell} \cdot\left(n m+n \cdot 2.076^{t}\right)\right)$-time algorithm and an $O(t \cdot \ell)$-vertex problem kernel for cost- $t$ packings.

(ii) For Feedback Arc Set in Tournaments, we show a $2^{O(\sqrt{(2 t+1) \ell})} \cdot n^{O(1)}$-time algorithm and an $O(t \cdot \ell)$-vertex problem kernel for cost- $t$ packings. 
(iii) For Cluster Editing, we show an $O\left(1.62^{(2 t+1) \cdot \ell}+n m+n \cdot 1.62^{t}\right)$-time algorithm and an $O(t \cdot \ell)$-vertex kernel for cost- $t$ packings, and a $4^{\ell} \cdot n^{O(1)}$-time algorithm for $P_{3}$-packings.

For the kernelization results, we need to assume that $t \in O(\log n)$ to guarantee polynomial running time of the data reduction.

Organization of this work. In Section 2, we introduce basic graph-theoretic notation and formally define fixed-parameter algorithms and problem kernelization. In Section 3, we present the general approach used in our algorithmic and data reduction results. In Section 4, we present our results regarding Triangle Deletion, in Section 5 regarding Feedback Arc Set in Tournaments, and in Section 6 regarding Cluster EDITING. Section 7 shows vertex and edge deletion problems that remain NP-hard for $\ell=0$, where $\ell$ is the number of modifications that are allowed in addition to a lower bound based on vertex-disjoint packings. We conclude with some open questions in Section 8.

\section{Preliminaries}

In this section, we introduce basic graph-theoretic notation and formally define fixedparameter algorithms and problem kernelization.

Notation. Unless stated otherwise, we consider undirected, simple, finite graphs $G=$ $(V, E)$, with a vertex set $V(G):=V$ and an edge set $E(G):=E \subseteq\left(\begin{array}{l}V \\ 2\end{array}\right):=\{\{u, v\} \mid u, v \in$ $V \wedge u \neq v\}$. Let $n:=|V(G)|$ denote the order of the graph and $m:=|E(G)|$ its number of edges. A set $S \subseteq\left(\begin{array}{c}V \\ 2\end{array}\right)$ is an edge modification set for $G$. For an edge modification set $S$ for $G$, let $G \triangle S:=(V,(E \backslash S) \cup(S \backslash E))$ denote the graph obtained by applying $S$ to $G$. If $S \subseteq E$, then $S$ is called an edge deletion set and we write $G \backslash S$ instead of $G \triangle S$. The open neighborhood of a vertex $v \in V$ is defined as $N_{G}(v):=\{u \in V \mid\{u, v\} \in E\}$. Also, for $V^{\prime} \subseteq V$, let $G\left[V^{\prime}\right]:=\left(V^{\prime}, E \cap\left(\begin{array}{c}V^{\prime} \\ 2\end{array}\right)\right)$ denote the subgraph of $G$ induced by $V^{\prime}$. A directed graph (or digraph) $G=(V, A)$ consists of a vertex set $V(G)$ and an $\operatorname{arc}$ set $A(G):=A \subseteq\left\{(u, v) \in V^{2} \mid u \neq v\right\}$. A tournament on $n$ vertices is a directed graph $(V, A)$ with $|V|=n$ such that, for each pair of distinct vertices $u$ and $v$, either $(u, v) \in A$ or $(v, u) \in A$.

Fixed-parameter algorithms. The idea in fixed-parameter algorithms is to accept the exponential running time that seems to be inevitable when exactly solving NP-hard problems, yet to confine it to some small problem-specific parameter. A problem is fixed-parameter tractable with respect to some parameter $k$ if there is a fixed-parameter algorithm solving any instance of size $n$ in $f(k) \cdot n^{O(1)}$ time. We will also say that a problem is fixed-parameter tractable with respect to some combined parameter " $k$ and $\ell$ " or " $(k, \ell)$ " if it is fixed-parameter tractable parameterized by $k+\ell$.

Fixed-parameter algorithms can efficiently solve instances in which the parameter $k$ is small, even if the input size $n$ is large. All vertex deletion, edge deletion, and edge modification problems for graph properties characterized by finite forbidden induced subgraphs are fixed-parameter tractable parameterized by the number of modifications $k[10]$. 
Problem kernelization. An important technique in fixed-parameter algorithmics is (problem) kernelization [31] - a formal approach of describing efficient and correct data reduction. A kernelization is an algorithm that given an instance $x$ with parameter $k$, yields an instance $x^{\prime}$ with parameter $k^{\prime}$ in time polynomial in $|x|+k$ such that $(x, k)$ is a yes-instance if and only if $\left(x^{\prime}, k^{\prime}\right)$ is a yes-instance, and if both $\left|x^{\prime}\right|$ and $k^{\prime}$ are bounded by some functions $g$ and $g^{\prime}$ in $k$, respectively. The function $g$ is referred to as the size of the problem kernel $\left(x^{\prime}, k^{\prime}\right)$. Kernelizations are commonly described by giving a set of data reduction rules which when applied to an instance $x$ of a problem yield an instance $x^{\prime}$. We say that a data reduction rule is correct if $x$ and $x^{\prime}$ are equivalent.

All vertex deletion problems for establishing graph properties characterized by a finite number of forbidden induced subgraphs have a problem kernel of size polynomial in the parameter $k$ of allowed modifications [30]. In contrast, many variants of $F$-FREE EDITING do not admit a problem kernel whose size is polynomial in $k[11,24,32]$.

\section{General Approach}

In this section, we describe the general approach of our fixed-parameter algorithms. Recall that $\tau(H)$ is the minimum number of edge modifications required to transform a graph $H$ into an $F$-free graph. We present fixed-parameter algorithms for three variants of $F$-FREe EDITING WITH CosT- $t$ PACKING parameterized by the combination of $t$ and $\ell:=k-h(\mathcal{H})$, where $h(\mathcal{H}):=\sum_{H \in \mathcal{H}} \tau(H)$. The idea behind the algorithms is to arrive at a classic win-win scenario [17] where we can either apply data reduction or show that the packing size $|\mathcal{H}|$ is bounded. This will allow us to bound $k$ in $t \cdot \ell$ for yes-instances and, thus, to apply known fixed-parameter algorithms for the parameter $k$ to obtain fixed-parameter tractability results for $(t, \ell)$.

More precisely, we show that, for each induced subgraph $H$ of $G$ in a given packing $\mathcal{H}$, we face essentially two situations. If there is an optimal solution for $H$ that is a subset of an optimal solution for $G$, then we can apply a data reduction rule. Otherwise, we find a certificate witnessing that $H$ itself needs to be solved suboptimally or that a vertex pair containing exactly one vertex from $H$ needs to be modified. We use the following terminology for these pairs.

Definition 3.1 (External vertex pairs and edges) A vertex pair $\{u, v\}$ is an external pair for a packing graph $H \in \mathcal{H}$ if exactly one of $u$ or $v$ is in $V(H)$, an edge is an external edge for $H \in \mathcal{H}$ if exactly one of its endpoints is in $H$.

Observe that every pair or edge is an external pair or edge for at most two packing graphs since the packing graphs are vertex-disjoint. Therefore, the modification of an external vertex pair can destroy at most two certificates. This is the main fact used in the proof of the following bound on $\ell$.

Lemma 3.2 Let $(G, \mathcal{H}, k)$ be an instance of $F$-Free Editing with Cost- $t$ Packing and let $S$ be a size- $k$ solution that contains, for each $H=(W, F) \in \mathcal{H}$,

(a) at least $\tau(H)+1$ vertex pairs from $\left(\begin{array}{c}W \\ 2\end{array}\right)$, or

(b) at least one external vertex pair $\{v, w\}$ for $H$. 
Then, $|\mathcal{H}| \leq 2 \ell$ and thus, $k \leq(2 t+1) \ell$.

Proof Denote by $\mathcal{H}_{a} \subseteq \mathcal{H}$ the set of all graphs in $\mathcal{H}$ that fulfill property (a) and let $p_{a}:=\left|\mathcal{H}_{a}\right|$. Let $\mathcal{H}_{b}:=\mathcal{H} \backslash \mathcal{H}_{a}$ denote the set containing the remaining packing graphs (fulfilling property (b)) and let $p_{b}:=\left|\mathcal{H}_{b}\right|$. Thus, $|\mathcal{H}|=p_{a}+p_{b}$. Furthermore, let $h_{a}:=\sum_{H \in \mathcal{H}_{a}} \tau(H)$ denote the lower bound obtained from the graphs in $\mathcal{H}_{a}$ and let $h_{b}:=h(\mathcal{H})-h_{a}$ denote the part of the lower bound obtained by the remaining graphs.

The packing graphs in $\mathcal{H}_{a}$ cause $h_{a}+p_{a}$ edge modifications inside of them. Similarly, the packing graphs in $\mathcal{H}_{b}$ cause at least $h_{b}$ edge modifications inside of them, and each packing graph $H \in \mathcal{H}_{b}$ additionally causes modification of at least one external vertex pair for $H$. Since every vertex pair is an external pair for at most two different packing graphs, at least $h_{b}+p_{b} / 2$ edge modifications are caused by the graphs in $\mathcal{H}_{b}$. This implies that

$$
\begin{aligned}
& & & \geq h_{a}+h_{b}+p_{a}+p_{b} / 2 \\
\Leftrightarrow & & k-h(\mathcal{H}) & \geq p_{a}+p_{b} / 2 \\
\Leftrightarrow & & 2 \ell & \geq 2 p_{a}+p_{b} \geq|\mathcal{H}| .
\end{aligned}
$$

Consequently, $k=\ell+h(\mathcal{H}) \leq \ell+t \cdot|\mathcal{H}| \leq \ell+t \cdot 2 \ell=(2 t+1) \ell$.

\section{Triangle Deletion}

In this section, we study Triangle Deletion, the problem of destroying all triangles $\left(K_{3} \mathrm{~s}\right)$ in a graph by at most $k$ edge deletions. In Section 4.1, we apply our framework from Section 3 to show that Triangle Deletion is fixed-parameter tractable parameterized above the lower bound given by a cost- $t$ packing. In Section 4.2 , we then show that parameterization above a lower bound given by edge-disjoint packings of triangles does not lead to fixed-parameter algorithms unless $\mathrm{P}=\mathrm{NP}$.

\subsection{A fixed-parameter algorithm for vertex-disjoint cost- $t$ packings}

Before presenting our new fixed-parameter tractability results for TRIANGLe Deletion, let us first summarize the known results concerning the (parameterized) complexity of Triangle Deletion. Triangle Deletion is NP-complete [47]. It allows for a trivial reduction to 3-Hitting Set since edge deletions do not create new triangles [23]. Combining this approach with the currently fastest known algorithms for 3-Hitring Set $[5,46]$ gives an algorithm for Triangle Deletion with running time $O\left(2.076^{k}+n m\right)$. Finally, Triangle Deletion admits a problem kernel with at most $6 k$ vertices [9]. We show that Triangle Deletion with Cost- $t$ Packing is fixed-parameter tractable with respect to the combination of $t$ and $\ell:=k-h(\mathcal{H})$. More precisely, we obtain a kernelization and a search tree algorithm. Both make crucial use of the following generic reduction rule for Triangle Deletion with Cost- $t$ Packing.

Reduction Rule 4.1 If there is an induced subgraph $H \in \mathcal{H}$ and a set $T \subseteq E(H)$ of $\tau(H)$ edges such that deleting $T$ destroys all triangles of $G$ that contain edges of $H$, then delete $T$ from $G, H$ from $\mathcal{H}$ and decrease $k$ by $\tau(H)$. 
Lemma 4.2 Reduction Rule 4.1 is correct.

Proof Let $(G, \mathcal{H}, k)$ be the instance to which Reduction Rule 4.1 is applied and let $\left(G^{\prime}, \mathcal{H} \backslash\{H\}, k-\tau(H)\right)$ with $G^{\prime}:=G \backslash T$ be the result. We show that $(G, \mathcal{H}, k)$ is a yes-instance if and only if $\left(G^{\prime}, \mathcal{H} \backslash\{H\}, k-\tau(H)\right)$ is.

First, let $S$ be a solution of size at most $k$ for $(G, \mathcal{H}, k)$. Let $S_{H}:=S \cap E(H)$ denote the set of edges of $S$ that destroy all triangles in $H$. By definition, $\left|S_{H}\right| \geq \tau(H)$. Since $S_{H} \subseteq E(H)$, only triangles containing at least one edge of $H$ are destroyed by deleting $S_{H}$. It follows that the set of triangles destroyed by $S_{H}$ is a subset of the triangles destroyed by $T$. Hence, $\left(S \backslash S_{H}\right) \cup T$ has size at most $k$ and clearly is a solution for $(G, \mathcal{H}, k)$ that contains all edges of $T$. Thus, $\left(S \backslash S_{H}\right)$ is a solution of size $k-\tau(H)$ for $G \backslash T=G^{\prime}$ and $\left(G^{\prime}, \mathcal{H} \backslash\{H\}, k-\tau(H)\right)$ is a yes-instance.

For the converse direction, let $S^{\prime}$ be a solution of size at most $k-\tau(H)$ for $\left(G^{\prime}, \mathcal{H} \backslash\right.$ $\{H\}, k-\tau(H))$. Since $T \subseteq E(H)$, it holds that every triangle contained in $G$ that does not contain any edge of $H$ is also a triangle in $G^{\prime}$. Thus, $S^{\prime}$ is a set of edges whose deletion in $G$ destroys all triangles that do not contain any edge of $H$. Since $T$ destroys all triangles containing an edge of $H$, we have that $T \cup S^{\prime}$ is a solution for $G$. Its size is $k$.

We now show that, if Reduction Rule 4.1 is not applicable to $H$, then we can find a certificate for this, which will allow us later to branch efficiently on the destruction of triangles:

Definition 4.3 (Certificate) A certificate for inapplicability of Reduction Rule 4.1 to an induced subgraph $H \in \mathcal{H}$ is a set $\mathcal{T}$ of triangles in $G$, each containing exactly one distinct edge of $H$, such that $|\mathcal{T}|=\tau(H)+1$ or $|\mathcal{T}| \leq \tau(H)$ and $\tau\left(H^{\prime}\right)>\tau(H)-|\mathcal{T}|$, where $H^{\prime}$ is the subgraph obtained from $H$ by deleting, for each triangle in $\mathcal{T}$, its edge shared with $H$.

Lemma 4.4 Let $\Gamma(G, k)$ be the time needed to compute a triangle-free deletion set of size at most $k$ in a graph $G$ if it exists.

In $O\left(n m+\sum_{H \in \mathcal{H}} \Gamma(H, t)\right)$ time, we can apply Reduction Rule 4.1 to all $H \in \mathcal{H}$ and output a certificate $\mathcal{T}$ if Reduction Rule 4.1 is inapplicable to some $H \in \mathcal{H}$.

In the statement of the lemma, we assume that $\Gamma$ is monotonically nondecreasing in the size of $G$ and in $k$. As described above, currently $O\left(2.076^{k}+|V(G)| \cdot|E(G)|\right)$ is the best known bound for $\Gamma(G, k)$.

Proof (of Lemma 4.4) First, in $O(\mathrm{~nm})$ time, we compute for all $H \in \mathcal{H}$ all triangles $\mathcal{T}$ that contain exactly one edge $e \in E(H)$. These edges are labeled in each $H \in \mathcal{H}$. Then, for each $H \in \mathcal{H}$, in $\Gamma(H, t)$ time we determine the size $\tau(H)$ of an optimal triangle-free deletion set for $H$. Let $t^{\prime}$ denote the number of labeled edges of $H$.

Case 1: $t^{\prime}>\tau(H)$. In this case, we return as certificate $\tau(H)+1$ triangles of $\mathcal{T}$, each containing a distinct of $\tau(H)+1$ arbitrary labeled edges.

Case 2: $t^{\prime} \leq \tau(H)$. Let $H^{\prime}$ denote the graph obtained from $H$ by deleting the labeled edges. All triangles of $G$ that contain at least one edge of $H$ either contain a labeled edge or they are contained in $H^{\prime}$. Thus, we now determine in $\Gamma\left(H^{\prime}, \tau(H)-t^{\prime}\right)$ time whether $H^{\prime}$ can be made triangle-free by $\tau(H)-t^{\prime}$ edge deletions. If this is the 
case, then the rule applies and the set $T$ consists of the solution for $H^{\prime}$ plus the deleted labeled edges. Otherwise, destroying all triangles that contain exactly one edge from $H$ leads to a solution which needs more than $\tau(H)$ edge deletions and thus the rule does not apply. In this case, we return the certificate $\mathcal{T}$ for this $H \in \mathcal{H}$.

The overall running time now follows from the monotonicity of $f$, from the fact that $|\mathcal{H}| \leq n$, and from the fact that one pass over $\mathcal{H}$ is sufficient since deleting edges in each $H$ does not produce new triangles and does not destroy triangles in any $H^{\prime} \neq H$.

Observe that Reduction Rule 4.1 never increases the parameter $\ell$ since we decrease both $k$ as well as the lower bound $h(\mathcal{H})$ by $\tau(H)$. After application of Reduction Rule 4.1, we can upper-bound the solution size $k$ in terms of $t$ and $\ell$, which allows us to transfer parameterized complexity results for the parameter $k$ to the combined parameter $(t, \ell)$.

Lemma 4.5 Let $(G, \mathcal{H}, k)$ be a yes-instance of Triangle Deletion with Cost- $t$ Packing such that Reduction Rule 4.1 is inapplicable. Then, $k \leq(2 t+1) \ell$.

Proof Since $(G, \mathcal{H}, k)$ is reduced with respect to Reduction Rule 4.1, for each graph $H=$ $(W, F)$ in $\mathcal{H}$, there is a set of edges between $W$ and $V \backslash W$ witnessing that every optimal solution for $H$ does not destroy all triangles containing at least one edge from $H$. Consider any optimal solution $S$. For each graph $H \in \mathcal{H}$, there are two possibilities: Either at least $\tau(H)+1$ edges inside $H$ are deleted by $S$, or at least one external edge of $H$ is deleted by $S$. Therefore, $S$ fulfills the condition of Lemma 3.2 and thus $k \leq(2 t+1) \ell$.

Theorem 4.6 Let $\Gamma(G, k)$ be the time used for computing a triangle-free deletion set of size at most $k$ in a graph $G$ if it exists. Then, Triangle Deletion with Cost- $t$ Packing

(i) can be solved in $O\left((2 t+3)^{\ell} \cdot\left(n m+\sum_{H \in \mathcal{H}} \Gamma(H, t)\right)\right)$ time, and

(ii) admits a problem kernel with at most $(12 t+6) \ell$ vertices that can be computed in $O\left(n m+\sum_{H \in \mathcal{H}} \Gamma(H, t)\right)$ time.

Proof We first prove (ii). To this end, let $(G=(V, E), \mathcal{H}, k)$ be the input instance. First, compute in $O\left(n m+\sum_{H \in \mathcal{H}} \Gamma(H, t)\right)$ time an instance that is reduced with respect to Reduction Rule 4.1. Afterwards, by Lemma 4.5, we can reject if $k>(2 t+1) \ell$. Otherwise, we apply the known kernelization algorithm for Triangle Deletion to the instance $(G, k)$ (that is, without $\mathcal{H})$. This kernelization produces in $O(m \sqrt{m})=$ $O(n m)$ time a problem kernel $\left(G^{\prime}, k^{\prime}\right)$ with at most $6 k \leq(12 t+6) \ell$ vertices and with $k^{\prime} \leq k$ [9]. Adding an empty packing gives an equivalent instance $\left(G^{\prime}, \emptyset, k^{\prime}\right)$ with parameter $\ell^{\prime}=k^{\prime} \leq(2 t+1) \ell$ of Triangle Deletion with Cost- $t$ Packing.

It remains to prove (i). To this end, first apply Reduction Rule 4.1 exhaustively in $O(n m+n \cdot \Gamma(H, t))$ time. Now, consider a reduced instance. If $\ell<0$, then we can reject the instance. Otherwise, consider the following two cases.

Case 1: $\mathcal{H}=\emptyset$. If $G$ is triangle-free, then we are done. Otherwise, pick an arbitrary triangle in $G$ and add it to $\mathcal{H}$.

Case 2: $\mathcal{H}$ contains a graph $H$. Since Reduction Rule 4.1 does not apply to $H$, there is a certificate $\mathcal{T}$ of $t^{\prime} \leq \tau(H)+1$ triangles, each containing exactly one distinct 
edge of $H$ such that deleting the edges of these triangles contained in $H$ produces a subgraph $H^{\prime}$ of $H$ that cannot be made triangle-free by $\tau(H)-t^{\prime}$ edge deletions. Thus, branch into the following $\left(2 t^{\prime}+1\right)$ cases: First, for each triangle $T \in \mathcal{T}$, create two cases, in each deleting a different one of the two edges of $T$ that are not in $H$. In the remaining case, delete the $t^{\prime}$ edges of $H$ and replace $H$ by $H^{\prime}$ in $\mathcal{H}$.

It remains to show the running time by bounding the search tree size. In Case 1 , no branching is performed and the parameter is decreased by at least one. In Case 2, the parameter value is decreased by one in each branch: in the first $2 t^{\prime}$ cases, an edge that is not contained in any packing graph is deleted. Thus, $k$ decreases by one while $h(\mathcal{H})$ remains unchanged. In the final case, the value of $k$ decreases by $t^{\prime}$ since this many edge deletions are performed. However, $\tau\left(H^{\prime}\right) \geq \tau(H)-t^{\prime}+1$. Hence, the lower bound $h(\mathcal{H})$ decreases by at most $t^{\prime}-1$ and thus the parameter $\ell$ decreases by at least one. Note that applying Reduction Rule 4.1 never increases the parameter. Hence, the depth of the search tree is at most $\ell$.

\section{Corollary 4.7 Triangle Deletion with Cost- $t$ Packing}

(i) can be solved in $O\left((2 t+3)^{\ell} \cdot\left(n m+n \cdot 2.076^{t}\right)\right.$ time, and

(ii) admits a problem kernel with at most $(12 t+6) \ell$ vertices that can be computed in $O\left(n m+n \cdot 2.076^{t}\right)$ time.

For the natural special case $t=1$, that is, for triangle packings, Theorem 4.6(i) immediately yields the following running time.

Corollary 4.8 Triangle Deletion with Triangle Packing is solvable in $O\left(5^{\ell} \mathrm{nm}\right)$ time.

\subsection{Hardness for edge-disjoint packing}

We complement the positive results of Theorem 4.6 by the following hardness result for the case of edge-disjoint triangle packings:

Theorem 4.9 Triangle Deletion is NP-hard even for $\ell:=k-|\mathcal{H}|=0$ if $\mathcal{H}$ is an edge-disjoint packing of triangles.

Theorem 4.9 shows that parameterizing Triangle Deletion over a lower bound given by edge-disjoint packings cannot lead to fixed-parameter algorithms unless $\mathrm{P}=\mathrm{NP}$. We prove Theorem 4.9 using a reduction from 3-SAT.

\section{Problem 4.10 (3-SAT)}

Input: A Boolean formula $\phi=C_{1} \wedge \ldots \wedge C_{m}$ in conjunctive normal form over variables $x_{1}, \ldots, x_{n}$ with at most three variables per clause.

Question: Does $\phi$ have a satisfying assignment?

Construction 4.11 Given a Boolean formula $\phi$, we create a graph $G$ and an edgedisjoint packing $\mathcal{H}$ of triangles such that $G$ can be made triangle-free by exactly $|\mathcal{H}|$ edge deletions if and only if there is a satisfying assignment for $\phi$. We assume that each clause of $\phi$ contains exactly three pairwise distinct variables. The construction is illustrated in Figure 4.1. 


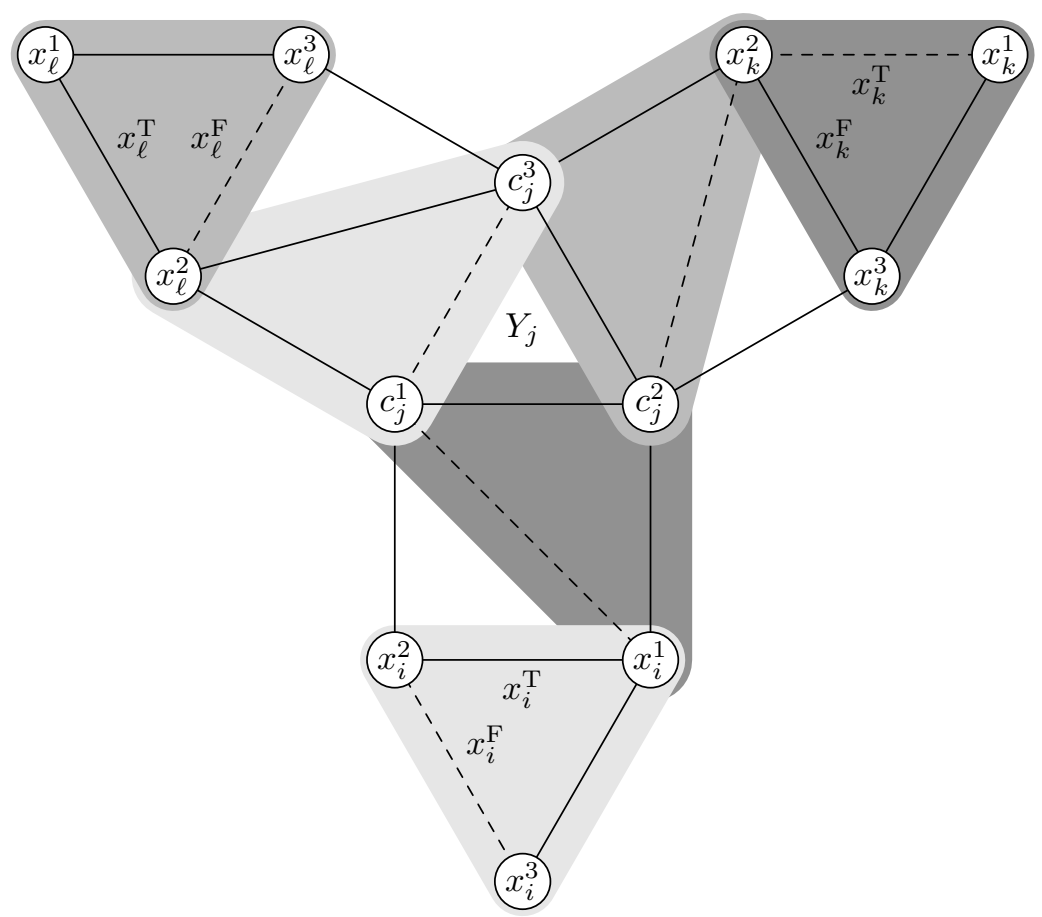

Fig. 4.1 Construction for a clause $C_{j}=\left(x_{i} \vee \neg x_{k} \vee \neg x_{\ell}\right)$. The triangles on a gray background are contained in the mutually edge-disjoint triangle packing $\mathcal{H}$. Deleting the dashed edges corresponds to setting $x_{i}$ and $x_{\ell}$ to false and $x_{k}$ to true, thus satisfying $C_{j}$. Note that it is impossible to destroy triangle $Y_{j}$ by $|\mathcal{H}|$ edge deletions if we delete $x_{i}^{\mathrm{F}}, x_{k}^{\mathrm{T}}$, and $x_{\ell}^{\mathrm{T}}$, which corresponds to the fact that clause $C_{j}$ cannot be satisfied by this variable assignment.

For each variable $x_{i}$ of $\phi$, create a triangle $X_{i}$ on the vertex set $\left\{x_{i}^{1}, x_{i}^{2}, x_{i}^{3}\right\}$ with two distinguished edges $x_{i}^{\mathrm{T}}:=\left\{x_{i}^{1}, x_{i}^{2}\right\}$ and $x_{i}^{\mathrm{F}}:=\left\{x_{i}^{2}, x_{i}^{3}\right\}$ and add $X_{i}$ to $\mathcal{H}$. For each clause $C_{j}=\left(l_{1}, l_{2}, l_{3}\right)$ of $\phi$, create a triangle $Y_{j}$ on the vertex set $\left\{c_{j}^{1}, c_{j}^{2}, c_{j}^{3}\right\}$ with three edges $c_{j}^{l_{1}}, c_{j}^{l_{2}}$, and $c_{j}^{l_{3}}$. Connect the clause gadget $Y_{j}$ to the variable gadgets as follows: If $l_{t}=x_{i}$, then connect the edge $c_{j}^{l_{t}}=:\{u, v\}$ to the edge $x_{i}^{\mathrm{T}}=\left\{x_{i}^{1}, x_{i}^{2}\right\}$ via two adjacent triangles $A_{i j}:=\left\{u, v, x_{i}^{1}\right\}$ and $B_{i j}:=\left\{v, x_{i}^{1}, x_{i}^{2}\right\}$ sharing the edge $\left\{v, x_{i}^{1}\right\}$. The triangle $A_{i j}$ is added to $\mathcal{H}$. If $l_{t}=\neg x_{i}$, then connect the edge $c_{j}^{l_{t}}=:\{u, v\}$ to the edge $x_{i}^{\mathrm{F}}=\left\{x_{i}^{2}, x_{i}^{3}\right\}$ via two adjacent triangles $A_{i j}:=\left\{u, v, x_{i}^{3}\right\}$ and $B_{i j}:=\left\{v, x_{i}^{2}, x_{i}^{3}\right\}$ sharing the edge $\left\{v, x_{i}^{3}\right\}$. The triangle $A_{i j}$ is added to $\mathcal{H}$.

Proof (of Theorem 4.9) First, observe that Construction 4.11 introduces no edges between distinct clause gadgets or distinct variable gadgets. Thus, under the assumption that each clause contains each variable at most once, the only triangles in the constructed graph are the $X_{i}$, the $Y_{j}$, the $A_{i j}$ and $B_{i j}$ for all variables $x_{i}$ and the incident clauses $C_{j}$.

Now, assume that $\phi$ allows for a satisfying assignment. We construct a set of edges $S$ of size $|\mathcal{H}|$ such that $G^{\prime}:=(V, E \backslash S)$ is triangle-free. For each variable $x_{i}$ that 
is true, add $x_{i}^{\mathrm{T}}$ to $S$. For each variable $x_{i}$ that is false, add $x_{i}^{\mathrm{F}}$ to $S$. By this choice, the triangle $X_{i}$ is destroyed in $G^{\prime}$ for each variable $x_{i}$. Additionally, for each clause $C_{j}$ and its true literals $l \in\left\{x_{i}, \neg x_{i}\right\}$, the triangle $B_{i j}$ is destroyed. To destroy $A_{i j}$, we add to $S$ the edge of $A_{i j}$ shared with $Y_{j}$, which also destroys the triangle $Y_{j}$. For each clause $C_{j}$ containing a false literal $l \in\left\{x_{i}, \neg x_{i}\right\}$, we destroy $B_{i j}$ and simultaneously $A_{i j}$ by adding to $S$ the edge of $A_{i j}$ shared with $B_{i j}$.

Conversely, assume that there is a set $S$ of size $|\mathcal{H}|$ such that $G^{\prime}=(V, E \backslash S)$ is triangle-free. We construct a satisfying assignment for $\phi$. First, observe that, since the triangles in $\mathcal{H}$ are pairwise edge-disjoint, $S$ contains exactly one edge of each triangle in $\mathcal{H}$. Thus, of each triangle $X_{i}$, at most one of the two edges $x_{i}^{\mathrm{F}}$ and $x_{i}^{\mathrm{T}}$ is contained in $S$. The set $S$ contains at least one edge $e$ of each $Y_{j}$. This edge is shared with a triangle $A_{i j}$. Since $A_{i j} \in \mathcal{H}$ and, with $e, S$ already contains one edge of $A_{i j}, S$ does not contain the edge shared between $A_{i j}$ and $B_{i j}$. Since $B_{i j} \notin \mathcal{H}, S$ has to contain an edge of $B_{i j}$ shared with another triangle in $\mathcal{H}$. If the clause $C_{j}$ contains $x_{i}$, then the only such edge is $x_{i}^{\mathrm{T}}$ and we set $x_{i}$ to true. If the clause $C_{j}$ contains $\neg x_{i}$, then the only such edge is $x_{i}^{\mathrm{F}}$ and we set $x_{i}$ to false. In both cases, clause $C_{j}$ is satisfied. Since at most one of $x_{i}^{\mathrm{T}}$ and $x_{i}^{\mathrm{F}}$ is in $S$, the value of each variable $x_{i}$ is well-defined.

\section{Feedback Arc Set in Tournaments}

In this section, we present a fixed-parameter algorithm and a problem kernel for FEEDBACK Arc SET In Tournaments parameterized above lower bounds of cost- $t$ packings.

In FeEdBack Arc Set in Tournaments, we are given a directed tournament graph $G$ as input and want to delete a minimum number of arcs to make the graph acyclic, that is, to destroy all directed cycles in $G$. Due to a well-known observation, one can also view Feedback Arc Set in Tournaments as an arc reversal problem: After deleting a minimum set of arcs to make the graph acyclic, adding the arc $(u, v)$ for every deleted $\operatorname{arc}(v, u)$ does not create any cycle. Since, in tournaments, every pair of vertices is connected by exactly one arc, it follows that that destroying cycles by edge deletions is equivalent to destroying them by arc reversals. Altogether, we arrive at the following problem definition.

\section{Problem 5.1 (Feedback Arc Set in Tournaments (FAST))}

Input: An $n$-vertex tournament $G=(V, A)$ and a natural number $k$.

Question: Does $G$ have a feedback arc set $S \subseteq A$, that is, a set $S$ such that reversing all $\operatorname{arcs}$ in $S$ yields an acyclic tournament, of size at most $k$ ?

FAST is NP-complete [1] but fixed-parameter tractable with respect to $k[2,14,16,20$, $27,42]$. The running time of the current best fixed-parameter algorithm is $2^{c \cdot \sqrt{k}}+n^{O(1)}$ where $c \leq 5.24$ [20]. Moreover, a problem kernel with $(2+\epsilon) k$ vertices for each constant $\epsilon>0$ is known [4] as well as a simpler $4 k$-vertex kernel [40]. It is wellknown that a tournament is acyclic if and only if it does not contain a directed triangle (a cycle on 3 vertices). Hence, the problem is to find a set of arcs whose reversal leaves no directed triangle in the tournament.

We show fixed-parameter tractability of FAST wITH Cost- $t$ PACKING parameterized by the combination of $t$ and $\ell:=k-h(\mathcal{H})$. Recall that $h(\mathcal{H}):=\sum_{H \in \mathcal{H}} \tau(H) \geq|\mathcal{H}|$, 
where $\tau(G)$ is the size of a minimum feedback arc set for a directed graph $G$. The approach is the same as for Triangle Deletion in Section 4, that is, we upper-bound the solution size $k$ in $t$ and $\ell$ and apply the fixed-parameter algorithm for $k$ [27]. Observe in this context that Lemma 3.2 is also correct if the input graphs are directed and if a solution contains arc reversals, since we observed arc reversals and deletions to be equivalent in the context of FAST. ${ }^{2}$ We use the following reduction rule for FAST analogous to Reduction Rule 4.1 for Triangle Deletion.

Reduction Rule 5.2 If there is a subtournament $H \in \mathcal{H}$ and a feedback arc set $T \subseteq$ $A(H)$ of size $\tau(H)$ such that reversing the arcs in $T$ leaves no directed triangles in $G$ containing $\operatorname{arcs}$ of $H$, then reverse the $\operatorname{arcs}$ in $T$, remove $H$ from $\mathcal{H}$, and decrease $k$ by $\tau(H)$.

Although Reduction Rule 5.2 is strikingly similar to Reduction Rule 4.1, its correctness proof is significantly more involved.

Lemma 5.3 Reduction Rule 5.2 is correct and, given the tournaments $G$ and $H$ it can be applied in $O\left(\left(\begin{array}{l}q \\ 2\end{array}\right)(n-q)+\Gamma(H, t)\right)$ time, where $q:=|V(H)|$ and $\Gamma(H, t)$ denotes the running time needed to compute a feedback arc set of size at most $t$ in $H$ if it exists.

Proof We first show correctness. Let $I^{\prime}:=\left(G^{\prime}, \mathcal{H} \backslash\{H\}, k-\tau(H)\right)$ be the instance created by Reduction Rule 5.2 from $I:=(G, \mathcal{H}, k)$ by reversing a subset $T$ of $\operatorname{arcs}$ of a subtournament $H \in \mathcal{H}$ of $G$. If $I^{\prime}$ is a yes-instance, then so is $I$ since $G^{\prime}$ is the graph $G$ with the $\tau(H)$ arcs in $T$ reversed and, thus, adding these arcs to a feedback arc set of size $k-\tau(H)$ for $G^{\prime}$ gives a feedback arc set of size $k$ for $G$. It remains to prove that if $I$ is a yes-instance, then so is $I^{\prime}$. To this end, we show that there is a minimum-size feedback arc set $S$ for $G$ with $T \subseteq S$.

Let $S$ be a minimum-size feedback arc set for $G=(V, E)$. This implies the existence of a linear ordering $\sigma_{S}=v_{1}, \ldots, v_{n}$ of the vertices $V$ such that there are $|S|$ backward arcs, that is, $\operatorname{arcs}\left(v_{i}, v_{j}\right) \in A$ such that $i>j$. Now, let $\sigma_{T}=w_{1}, \ldots, w_{|W|}$ be the ordering of the vertices of $H=(W, F)$ corresponding to the local solution $T$ for $H$ with $\tau(H)$ backward arcs. Let $N^{+}(w):=\{(w, v) \in A \mid v \in V \backslash W\}$ denote the out-neighbors in $V \backslash W$ of a vertex $w \in W$. Analogously, $N^{-}(w):=\{(v, w) \in A \mid v \in$ $V \backslash W\}$ denotes the set of in-neighbors. By the assumption of the rule, for all $i<j$,

$$
N^{+}\left(w_{j}\right) \subseteq N^{+}\left(w_{i}\right) \text { and } N^{-}\left(w_{i}\right) \subseteq N^{-}\left(w_{j}\right)
$$

holds since otherwise, after reversing the arcs in $T$, there exists a directed cycle containing an arc of $H$ (because the $\operatorname{arc}\left(w_{i}, w_{j}\right)$ is present).

If the vertices of $W$ appear in $\sigma_{S}$ in the same relative order as in $\sigma_{T}$, then we have $T \subseteq S$ and we are done. Otherwise, we show that we can swap the positions of vertices of $W$ in $\sigma_{S}$ so that their relative order is the same as in $\sigma_{T}$ without increasing the number of backward arcs.

First, note that the number of backward arcs between vertices in $V \backslash W$ does not change when only swapping positions of vertices in $W$. Also, by assumption, the number of backward arcs between vertices in $W$ in any ordering is at least $\tau(H)$,

\footnotetext{
${ }^{2}$ For directed input graphs, we use the term external arc instead of external edge.
} 
whereas it is exactly $\tau(H)$ when ordering them according to $\sigma_{T}$. Thus, it remains to show that the number of backward arcs between vertices in $W$ and $V \backslash W$ is not increased. To this end, consider a series of swaps of pairs of vertices $w_{j}$ and $w_{i}$ such that $i<j$, where $w_{j}$ appears before $w_{i}$ in $\sigma_{S}$, reordering the vertices in $W$ according to $\sigma_{T}$. Let $Y$ denote the set of all vertices that lie between $w_{j}$ and $w_{i}$ in $\sigma_{S}$. Note that swapping $w_{j}$ and $w_{i}$ removes the backward arcs from $w_{i}$ to the vertices in $N^{+}\left(w_{i}\right) \cap Y$ and the backward arcs from vertices in $N^{-}\left(w_{j}\right) \cap Y$ to $w_{j}$, whereas it introduces new backward arcs from $w_{j}$ to $N^{+}\left(w_{j}\right) \cap Y$ and from $N^{-}\left(w_{i}\right) \cap Y$ to $w_{i}$. However, by the inclusions in (1), it follows that the overall number of backward arcs does not increase in each swap. Hence, the overall number of backward arcs is not increased by repositioning the vertices in $W$ according to $\sigma_{T}$. It follows that there is an optimal solution containing $T$.

It remains to show the running time. First, in $\Gamma(H, \tau(H))$ time, we compute the size $\tau(H)$ of an optimal feedback arc set for $H=(W, F)$. Now, for each $\operatorname{arc}(u, v) \in F$, we check whether there is a vertex $w \in V \backslash W$ that forms a directed triangle with $u$ and $v$. If such a vertex exists, then we reverse the $\operatorname{arc}(u, v)$. If this arc reversal introduces a new directed triangle with another vertex from $V \backslash W$, then the rule does not apply. Overall, this procedure requires $O(|F| \cdot(n-|W|))$ time. Let $T^{*}$ denote the set of arcs that are reversed in this process. Clearly, if $\left|T^{*}\right|>\tau(H)$, then the rule does not apply. Otherwise, let $H^{\prime}$ denote the graph obtained from $H$ by reversing the $\operatorname{arcs}$ in $T^{*}$ and observe that each remaining directed triangle of $G$ that contains at least one arc of $H^{\prime}$ is contained in $H^{\prime}$. Thus, we now compute whether $H^{\prime}$ has a feedback arc set $T^{\prime}$ of size $\tau(H)-\left|T^{*}\right|$ in $\Gamma\left(H^{\prime}, \tau(H)-\left|T^{*}\right|\right)$ time. If this is the case, then the rule applies and we set $T:=T^{\prime} \cup T^{*}$ (note that $T^{\prime} \cap T^{*}=\emptyset$, since otherwise $|T|<\tau(H)$, which is not possible by definition of $\tau(H)$ ). Otherwise, removing all directed triangles that contain at least one arc from $H$ requires more than $\tau(H)$ arc reversals and thus the rule does not apply.

Exhaustive application of Reduction Rule 5.2 allows us to show that $k \leq(2 t+1) \ell$ holds for any yes-instance (analogous to Lemma 4.5).

Lemma 5.4 Let $(G, \mathcal{H}, k)$ be a yes-instance of Feedback Arc Set in Tournaments with Cost- $t$ Packing such that Reduction Rule 5.2 cannot be applied to any tournament in $\mathcal{H}$. Then, $k \leq(2 t+1) \ell$.

Proof Since Reduction Rule 5.2 cannot be applied to any tournament in $\mathcal{H}$, for each tournament $H=(W, F)$ in $\mathcal{H}$, there is a set of arcs between $W$ and $V \backslash W$ that witness that no optimal feedback arc set for $H$ removes all directed triangles containing at least one arc from $F$.

Now, for any optimal solution, there are two possibilities for each packing tournament $H \in \mathcal{H}$ :

(a) at least $\tau(H)+1 \operatorname{arcs}$ in $H$ are reversed, or

(b) at least one external arc of $H$ is reversed.

Therefore, $S$ fulfills the condition of Lemma 3.2 and, thus, $|\mathcal{H}| \leq 2 \ell$.

The bound on $k$ yields the following two fixed-parameter tractability results. 
Theorem 5.5 Let $\Gamma(G, k)$ be the running time used for finding a minimum feedback arc set of size at most $k$ for a given tournament $G$ if it exists. Then, FeEdBack Arc SET In Tournaments with Cost- $t$ Packing

(i) is solvable in $\Gamma(G,(2 t+1) \ell)+n^{O(1)}+\sum_{H \in \mathcal{H}} \Gamma(H, t)$ time, and

(ii) admits a problem kernel with at most $(8 t+4) \ell$ vertices computable in $n^{O(1)}+$ $\sum_{H \in \mathcal{H}} \Gamma(H, t)$ time.

Proof (i) Given $(G, \mathcal{H}, k)$, we first apply Reduction Rule 5.2 for each $H \in \mathcal{H}$. This application can be performed in $O\left(|\mathcal{H}| n^{3}+\sum_{H \in \mathcal{H}} \Gamma(H, t)\right)$ time by Lemma 5.3 since $|\mathcal{H}| \leq n$. One pass of this rule is sufficient to obtain an instance that is reduced: reversing arcs in some $H \in \mathcal{H}$ does not remove any directed triangles containing arcs of any other $H^{\prime} \in \mathcal{H}$ with $H^{\prime} \neq H$. By Lemma 5.4, we can then reject the instance if $k>(2 t+1) \ell$. Otherwise, we can find a solution in $\Gamma(G,(2 t+1) \ell)$ time.

(ii) First, we apply Reduction Rule 5.2 once for each $H \in \mathcal{H}$ in $O\left(|\mathcal{H}| n^{3}+\right.$ $\left.\sum_{H \in \mathcal{H}} \Gamma(H, t)\right)$ time. After one pass of this rule, the instance is reduced since reversing arcs in some $H \in \mathcal{H}$ does not remove any directed triangles containing arcs of any other $H^{\prime} \in \mathcal{H}$ with $H^{\prime} \neq H$. Afterwards, by Lemma 5.4, we can reject if $k>(2 t+1) \ell$. Otherwise, we apply the kernelization algorithm for FAST by Paul et al [40] to the instance $(G, k)$ to obtain an equivalent instance $\left(G^{\prime}, k^{\prime}\right)$ with at most $4 k \leq(8 t+4) \ell$ vertices and a solution size $k^{\prime} \leq k$. Hence, $\left(G^{\prime}, \emptyset, k^{\prime}\right)$ is our problem kernel with parameter $\ell^{\prime}=k^{\prime} \leq(2 t+1) \ell$ of Feedback Arc Set in Tournaments with Cost- $t$ PACKING.

In Theorem 5.5, we again assume that $\Gamma$ is monotonically nondecreasing in both the size of $G$ and in $k$. As mentioned earlier, $2^{O(\sqrt{k})}+|V(G)|^{O(1)}$ is the currently best known running time for $\Gamma(G, k)$ [27].

Corollary 5.6 Feedback Arc Set in Tournaments with Cost- $t$ Packing

(i) can be solved in $2^{O(\sqrt{(2 t+1) \ell})}+n^{O(1)}+n 2^{O(\sqrt{t})}$ time, and

(ii) admits a problem kernel with at most $(8 t+4) \ell$ vertices computable in $n^{O(1)}+$ $n 2^{O(\sqrt{t})}$ time.

\section{Cluster Editing}

We finally apply our framework from Section 3 to CLuster EDITING, a well-studied edge modification problem in parameterized complexity [7, 12, 21, 28].

Problem 6.1 (Cluster Editing)

Input: A graph $G=(V, E)$ and a natural number $k$.

Question: Is there an edge modification set $S \subseteq\left(\begin{array}{l}V \\ 2\end{array}\right)$ of size at most $k$ such that $G \triangle S$ is a cluster graph, that is, a disjoint union of cliques?

A graph is a cluster graph if and only if it is $P_{3}$-free [45]. Thus, Cluster Editing is the problem of destroying all $P_{3}$ s by few edge modifications. For brevity, we refer to the connected components of a cluster graph (which are cliques) and to their vertex sets as clusters. The currently fastest algorithm for CLusTER EDITING parameterized by 

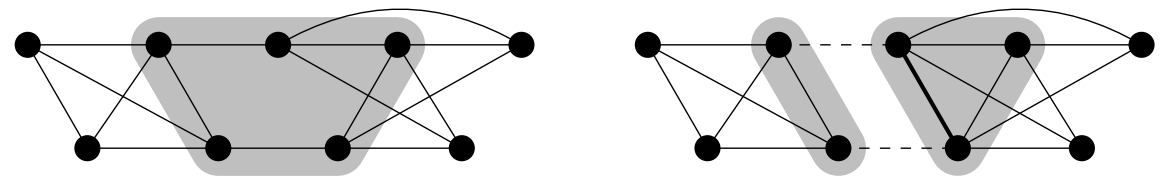

Fig. 6.1 An illustration of Reduction Rule 6.2. Left: An induced subgraph $H$ (highlighted by the gray background) fulfilling the conditions of Reduction Rule 6.2. Right: The result of applying Reduction Rule 6.2. The two clusters in $G[W]$ produced by the optimal solution for $H$ are highlighted by a gray background.

the solution size $k$ runs in $O\left(1.62^{k}+n+m\right)$ time [7]. Assuming the exponential-time hypothesis, Cluster Editing cannot be solved in $2^{o(k)} \cdot n^{O(1)}$ time [21, 28]. Cluster EDITING admits a problem kernel with at most $2 k$ vertices [12].

First, in Section 6.1, we present a fixed-parameter algorithm and problem kernel for CLuster Editing parameterized above lower bounds given by cost- $t$ packings. Then, in Section 6.2, we present a faster fixed-parameter algorithm for lower bounds given by vertex-disjoint packings of $P_{3}$ s.

\subsection{A fixed-parameter algorithm for vertex-disjoint cost- $t$ packings}

Several kernelizations for CLuSTER EDITING are based on the following observation: If $G$ contains a clique such that all vertices in this clique have the same closed neighborhood, then there is an optimal solution that puts these vertices into the same cluster $[12,25,41]$. This implies that the edges of this clique are never deleted. The following rule is based on a generalization of this observation.

Reduction Rule 6.2 If $G=(V, E)$ contains an induced subgraph $H=(W, F)$ having an optimal solution $S$ of size $\tau(H)$ such that, for all vertices $u, v \in W$,

- $N_{G}(v) \backslash W=N_{G}(u) \backslash W$ if $u$ and $v$ are in the same cluster of $H \triangle S$, and

- $N_{G}(v) \cap N_{G}(u) \subseteq W$ otherwise,

then replace $G$ by $G \triangle S$, remove $H$ from $\mathcal{H}$, and decrease $k$ by $\tau(H)$.

An example of Reduction Rule 6.2 is presented in Figure 6.1.

Lemma 6.3 Reduction Rule 6.2 is correct.

Proof Let $I^{\prime}:=\left(G \triangle S, \mathcal{H}^{\prime}, k-\tau(H)\right)$ be the instance obtained by applying Reduction Rule 6.2 to $I:=(G, \mathcal{H}, k)$ for some induced subgraph $H=(W, F)$ of $G=(V, E)$. If $I^{\prime}$ is a yes-instance, then so is $I$ : adding the $\tau(H)$ edges in $S$ to any solution $S^{\prime}$ of size $k-\tau(H)$ for $G \triangle S$ gives a solution of size $k$ for $G$ since $S \cap S^{\prime}=\emptyset$ and, thus, $G \triangle\left(S \cup S^{\prime}\right)=(G \triangle S) \triangle S^{\prime}$. It remains to prove that if $I$ is a yes-instance, then so is $I^{\prime}$. To this end, we show that $(G, \mathcal{H}, k)$ has an optimal solution $S^{\prime}$ such that $S \subseteq S^{\prime}$.

For convenience, let $X:=V \backslash W$ denote the set of vertices not in $W$. Let $S^{*}$ be any optimal solution for $G$, and denote by $G^{*}:=G \triangle S^{*}$ the cluster graph produced by $S^{*}$. We show how to transform $S^{*}$ into an optimal solution $S^{\prime} \supseteq S$. To this end, partition $S^{*}$ as follows: 
- $S_{1}^{*}:=\left\{\{u, v\} \in S^{*} \mid u, v \in X\right\}$ containing all edge modifications outside of $H$,

$-S_{2}^{*}:=\left\{\{u, v\} \in S^{*} \mid u \in X \wedge v \in W\right\}$ containing the edge modifications between $H$ and the rest of $G$, and

$-S_{3}^{*}:=\left\{\{u, v\} \in S^{*} \mid u, v \in W\right\}$.

Moreover, let $W_{\text {ext }}$ be the vertices of $W$ that have at least one neighbor in $X$ and let $W_{\text {int }}:=W \backslash W_{\text {ext }}$. Consider the following equivalence relation $\sim$ on $W_{\text {ext }}$ : two vertices $u, v \in W_{\text {ext }}$ are equivalent with respect to $\sim$ if and only if $N_{G}(u) \cap X=N_{G}(v) \cap X$. For each vertex $u \in W_{\text {ext }}$, let $[u]$ denote the equivalence class of $u$ in $\sim$.

Fix within each equivalence class $[u]$ of $\sim$ an arbitrary vertex that is incident to a minimum number of edge modifications in $S_{2}^{*}$ and, for each vertex $u$, denote this vertex by $\tilde{u}$. Furthermore, for each cluster $K$ of $G^{*}$ containing some vertices of $X$ and some vertices of $W_{\text {ext }}$, fix an arbitrary vertex of $W_{\text {ext }}$ that has in $G$ a maximum number of neighbors in $K \cap X$; denote this vertex by $u_{K}$. Finally, call a vertex $u \in W_{\text {ext }}$ good if there is a cluster $K$ such that $\tilde{u}=u_{K}$. Now consider the edge modification set $S^{\prime}:=S_{1}^{*} \cup \tilde{S} \cup S$, where

$$
\begin{aligned}
\tilde{S}: & =\left\{\{u, v\} \mid u \text { is good and }\{\tilde{u}, v\} \in S_{2}^{*}\right\} \cup \\
& \cup\left\{\{u, v\} \mid u \text { is not good and } v \in N_{G}(u) \cap X\right\} .
\end{aligned}
$$

Informally, the modifications in $\tilde{S}$ consider $\tilde{u}$ to determine how to treat all vertices in the equivalence class $[u]$. If, among the vertices of $W_{\text {ext }}, \tilde{u}$ has the most neighbors in its cluster in $G^{*}$, then all vertices of $[u]$ are treated like $\tilde{u}$. Otherwise, all edges between vertices in $[u]$ and $X$ are deleted.

We first show that $S^{\prime}$ is a solution, that is, $G^{\prime}:=G \triangle S^{\prime}$ is a cluster graph: First, $G^{\prime}[X]=G^{*}[X]$ is a cluster graph. Second, $G^{\prime}[W]=H \triangle S$ and thus it is a cluster graph. Third, every vertex $u \in W_{\text {int }}$ is contained in a cluster that is a subset of $W_{\text {int }}$ in $G^{\prime}:$ In $G$, there are no edges between $W_{\text {int }}$ and $X$, no edges between $W_{\text {int }}$ and $X$ are added by $\tilde{S}$, and by the first condition on $S$, no vertex of $W_{\text {int }}$ is in the same cluster of $H \triangle S$ as a vertex from $W_{\text {ext }}$. This implies that the connected component of each vertex $u \in W_{\text {int }}$ is completely contained in $W$ and thus it is a clique since $G^{\prime}[W]$ is a cluster graph. Finally, consider any equivalence class $[u]$ of $W_{\text {ext }}$. By the condition of Reduction Rule 6.2, $[u]$ is contained in a cluster in $H \triangle S$. Now, if all vertices of $[u]$ are good, then there is a cluster $K$ in $G^{\prime}[X]$ such that in $G^{\prime}$ all vertices of $[u]$ are adjacent to all vertices of $K$. Otherwise, no vertex of $[u]$ is good and thus, no vertex of $[u]$ is adjacent to any vertex of $X$ in $G^{\prime}$. Finally, if a cluster $K$ of $G^{\prime}[X]$ has neighbors in $W$, then these edges are only to the vertex set $\left[u_{K}\right]$. Thus, every vertex in $K$ has neighbors in at most one cluster of $H \triangle S$. Altogether this shows that $G^{\prime}$ is a cluster graph.

It remains to show that $\left|S^{\prime}\right| \leq\left|S^{*}\right|$. First, $S_{1}^{*}$ is a subset of $S^{*}$ and of $S^{\prime}$. Second, $|S| \leq\left|S_{3}^{*}\right|$ since $S$ is an optimal solution for $H$. Thus, it remains to show $|\tilde{S}| \leq\left|S_{2}^{*}\right|$. Since the vertices of $W_{\text {int }}$ are not incident to any edge modifications in $\tilde{S}$, we may prove this inequality by showing, for each vertex $u \in W_{\mathrm{ext}}$, that

$$
|\{e \in \tilde{S} \mid u \in e\}| \leq\left|\left\{e \in S_{2}^{*} \mid u \in e\right\}\right| .
$$

If $u$ is good, then the number of edge modifications incident with $u$ in $\tilde{S}$ is the same as the number of edge modifications incident with $\tilde{u}$ in $S_{2}^{*}$, because $u$ and $\tilde{u}$ have the 
same neighborhood in $X$ by the condition of the rule. By the choice of $\tilde{u}$, this is at most as large as the number of edge modifications incident with $u$ in $S_{2}^{*}$ and the claim holds.

Otherwise, all edges between $u$ and $X$ are deleted by $\tilde{S}$. Let $K$ denote the cluster in $G \triangle S^{*}$ containing $\tilde{u}$. Since $u$ is not good, there is a vertex $w$ that is not in $[u]$ such that $w$ has at least as many neighbors in $K \cap X$ as $\tilde{u}$. By the condition of Reduction Rule 6.2 and since $[\tilde{u}] \neq[w]$, the neighborhoods of these two vertices in $X$ are disjoint, that is, $\left(N_{G}(\tilde{u}) \cap X\right) \cap\left(N_{G}(w) \cap X\right)=\emptyset$. Since $w$ has at least as many neighbors in $K \cap X$ as $\tilde{u}$, this means that

$$
\left|N_{G}(\tilde{u}) \cap K \cap X\right| \leq|K \cap X| / 2 .
$$

Now, observe that $S_{2}^{*}$ contains an edge insertion between $\tilde{u}$ and each nonneighbor of $\tilde{u}$ in $K \cap X$. Thus, at least $|K \cap X| / 2$ edges between $\tilde{u}$ and $K \cap X$ are inserted by $S_{2}^{*}$. Moreover, $S_{2}^{*}$ contains an edge deletion between $\tilde{u}$ and each neighbor of $\tilde{u}$ in $X \backslash K$. Altogether, this implies

$$
\left|\left\{e \in S_{2}^{*}: \tilde{u} \in e\right\}\right| \geq|K \cap X| / 2+\left|N_{G}(\tilde{u}) \cap(X \backslash K)\right| \geq\left|N_{G}(\tilde{u}) \cap X\right| .
$$

Therefore, the number of edge modifications incident with $u$ in $\tilde{S}$ (this is exactly the number of edges between $u$ and $X$ ) is at most as large as the number of edge modifications incident with $\tilde{u}$ in $S_{2}^{*}$. By the choice of $\tilde{u}$, this implies the claim.

It remains to analyze the running time for applying Reduction Rule 6.2.

Lemma 6.4 Let $\Gamma(G, k)$ be the running time used for finding an optimal solution of size at most $k$ in a graph $G$ if it exists. in $\mathcal{H}$.

Then, in $O\left(m+\sum_{H \in \mathcal{H}} \Gamma(H, t)\right)$ time, we can apply Reduction Rule 6.2 to all graphs

Here, we assume that $\Gamma$ is monotonically nondecreasing in $k$ and polynomial in the size of $G$. Currently, $O\left(1.62^{k}+|V(G)|+|E(G)|\right)$ is the best known bound for $\Gamma(G, k)[7]$.

Proof We first show that the rule can be applied in $O\left(|W|+\sum_{w \in W} \operatorname{deg}_{G}(w)+\Gamma(H, t)\right)$ time to an arbitrary graph $H=(W, F) \in \mathcal{H}$. For convenience, denote $X:=V \backslash W$.

First, observe that a necessary condition for the rule is that, for each pair of vertices $u$ and $v$ in $H$, their neighborhoods in $X$ are the same or disjoint. This can be checked in $O\left(|W|+\sum_{w \in W} \operatorname{deg}_{G}(w)\right)$ time as follows. First, build the bipartite graph with parts $W$ and $N(W)$ and those edges between the vertex sets that are also edges of $G$ (equivalently, $G[W \cup N(W)]$ minus the edges with both endpoints in $W$ or both endpoints in $N(W)$ ). This bipartite graph is a disjoint union of complete bipartite graphs if and only if above condition is fulfilled. Thus, we check in $O(|W|+|F|)$ time, whether the graph is a disjoint union of complete bipartite graphs. If not, then the rule does not apply. Otherwise, in the created bipartite graph, we compute in $O(|W|+|F|)$ time the groups of vertices of $W$ whose neighborhood is the same. Afterwards, we can check in $O(1)$ time whether $u$ and $v$ have the same neighborhood in $X$ in $G$ by checking whether they belong to the same group. We now compute the set $S^{\prime}$ of edge modifications that is already determined by the conditions of the rule: If $v$ and $w$ are nonadjacent and have the same nonempty neighborhood in $X$, then the edge $\{v, w\}$ needs to be 
inserted and is thus added to $S^{\prime}$. Similarly, if $v$ and $w$ are adjacent and have different neighborhoods in $X$, then $\{v, w\}$ needs to be deleted and is thus added to $S^{\prime}$. Observe that if $S^{\prime}$ is a subset of an optimal solution $S$ for $H$, then $|S| \leq|F|$. Hence, at most $|F|$ edges are added to $S^{\prime}$. Since we already computed the groups of vertices that have the same or disjoint neighborhoods, we can thus compute $S^{\prime}$ in $O(|W|+|F|)$ time.

Let $W_{\text {ext }}$ denote the vertices of $W$ that have at least one neighbor in $N(W)$ and let $W_{\text {int }}:=W \backslash W_{\text {ext }}$. Note that after applying $S^{\prime}$, that is, in $H \triangle S^{\prime}$, the vertices of $W_{\text {ext }}$ are in separate clusters: $\left(H \triangle S^{\prime}\right)\left[W_{\text {ext }}\right]$ is a cluster graph and there are no edges between $W_{\text {ext }}$ and $W_{\text {int }}$ in $H \triangle S^{\prime}$. Thus, to determine whether $S^{\prime}$ can be extended to an optimal solution $S$ for $H$ that fulfills the condition of the rule, we compute an optimal solution of $H\left[W_{\text {int }}\right]$ in $\Gamma\left(H\left[W_{\text {int }}\right], \tau(H)-\left|S^{\prime}\right|\right)$ time. Since $\left|H\left[W_{\text {int }}\right]\right| \leq|H|$ and $\tau(H)-\left|S^{\prime}\right|<t$, this can be done in $\Gamma(H, t)$ time. The size of the resulting solution $S$ is compared with the size of an optimal solution of $H$, which can also be computed in $\Gamma(H, t)$ time.

It remains to show that Reduction Rule 6.2 can be applied to all graphs within the claimed running time. For each graph $H=(W, F) \in \mathcal{H}$, we can check in $O(|W|+$ $\left.\sum_{w \in W} \operatorname{deg}_{G}(w)+\Gamma(H, t)\right)$ time whether Reduction Rule 6.2 applies. If yes, then we can apply the rule in $O(|F|)$ time by modifying at most $|F|$ edges. Summing up over all graphs in $\mathcal{H}$ gives the claimed running time.

Observe that since $k$ is decreased by $\tau(H)$, the parameter $\ell$ does not increase when Reduction Rule 6.2 is applied. As for the previous problems, applying the rule to each $H \in \mathcal{H}$ is sufficient for bounding $k$ in $t$ and $\ell$ and thus, for transferring known fixed-parameter tractability results for the parameter $k$ to the combined parameter $(t, \ell)$.

Lemma 6.5 Let $(G, \mathcal{H}, k)$ be a yes-instance of Cluster Editing with Cost- $t$ Packing such that Reduction Rule 6.2 does not apply to any $H \in \mathcal{H}$. Then, $k \leq(2 t+1) \ell$.

Proof Since the instance is reduced with respect to Reduction Rule 6.2, for each $H=$ $(W, F)$ in $\mathcal{H}$ and each size- $\tau(H)$ solution $S$ for $H$, either the vertices of some cluster have different neighborhoods in $V \backslash W$ or two vertices of two distinct clusters have a common neighbor outside of $W$.

Now, fix an arbitrary optimal solution $S$ for $G$. By the observation above, there are the following two possibilities for how $S$ modifies each $H \in \mathcal{H}$ :

(a) more than $\tau(H)$ vertex pairs of $H$ are modified by $S$, or

(b) at least one external vertex pair for $H$ is modified.

Therefore, $S$ fulfills the condition of Lemma 3.2 and thus $k \leq(2 t+1) \ell$.

Theorem 6.6 Let $\Gamma(G, k)$ be the running time used for finding an optimal solution of size at most $k$ in a graph $G$ if it exists. Then, Cluster Editing with Cost- $t$ Packing

(i) is solvable in $O\left(\Gamma(G,(2 t+1) \ell)+n m+\sum_{H \in \mathcal{H}} \Gamma(H, t)\right)$ time and

(ii) admits a problem kernel with at most $(4 t+2) \ell$ vertices, which can be computed in $O\left(n m+\sum_{H \in \mathcal{H}} \Gamma(H, t)\right)$ time.

Proof (ii) First, apply Reduction Rule 6.2 exhaustively in $O\left(m+\sum_{H \in \mathcal{H}} \Gamma(H, t)\right)$ time. Then, by Lemma 6.5, we can either return "no" or have $k \leq(2 t+1) \ell$. In the latter 
case, we apply a kernelization algorithm for CLuster EDITING to the instance $(G, k)$ (that is, without $\mathcal{H}$ ), which produces, in $O(n m)$ time, a problem kernel $\left(G^{\prime}, k^{\prime}\right)$ with at most $2 k \leq(4 t+2) \ell$ vertices and with $k^{\prime} \leq k$ [12]. Adding an empty packing gives an equivalent instance $\left(G^{\prime}, \emptyset, k^{\prime}\right)$ with parameter $\ell^{\prime}=k^{\prime}$ of CLuster Editing With Cost- $t$ PACKING.

(i) First, apply the kernelization. Then, by Lemma 6.5, we can either return "no" or have $k \leq(2 t+1) \ell$. We can now apply the algorithm for Cluster EdITING that runs in $\Gamma(G,(2 t+1) \ell)$ time.

By plugging in the best known bound for $\Gamma(G, k)$, we obtain the following.

\section{Corollary 6.7 Cluster Editing with Cost- $t$ Packing}

(i) can be solved in $O\left(1.62^{(2 t+1) \cdot \ell}+n m+n \cdot 1.62^{t}\right)$ time, and

(ii) admits a problem kernel with at most $(4 t+2) \ell$ vertices that can be computed in $O\left(n m+n \cdot 1.62^{t}\right)$ time.

\subsection{A Search Tree Algorithm for $P_{3}$-Packings}

For Cluster Editing with $P_{3}$-Packing, the generic algorithm based on Reduction Rule 6.2 (with $t=1$ ) using the currently best running time for CLuster EdITING leads to a running time of $O\left(4.26^{\ell}+n+m\right)$. We now show an algorithm that runs in $O\left(4^{\ell} \cdot \ell^{3}+n+m\right)$ time. The algorithm is based on two special cases of Reduction Rule 6.2, one further reduction rule and a corresponding branching algorithm.

The analysis of the search tree size is done by considering branching vectors and their corresponding branching number. The components of a branching vector denote the decrease of the parameter in each recursive branch. The branching number depends only on the branching vector, the largest branching number gives the base in the upper bound on the search tree size; for further details refer to the relevant monographs [19, 39].

We use the following special cases of Reduction Rule 6.2. In both cases, $\mathrm{H}$ is a $P_{3}$; the correctness is directly implied by Lemma 6.4. In the first rule, adding an edge is a solution which fulfills the condition of Reduction Rule 6.2. For convenience, we denote by $u v w$ a $P_{3}$ on the vertices $u, v$, and $w$, where $v$ is the degree-two vertex.

Reduction Rule 6.8 If $G$ contains a $P_{3} u v w$ such that $N(u) \backslash\{u, v, w\}=N(v) \backslash$ $\{u, v, w\}=N(w) \backslash\{u, v, w\}$, then insert $\{u, w\}$ and decrease $k$ by one.

In the second rule, deleting an edge gives such a solution.

Reduction Rule 6.9 If $G$ contains a $P_{3} u v w$ such that $N(u) \backslash\{u, v, w\}=N(v) \backslash\{u, v, w\}$ and $N(u) \cap N(w)=\{v\}$, then delete $\{v, w\}$ and decrease $k$ by one.

The third rule is crucial for showing an improved running time.

Reduction Rule 6.10 If $G$ contains a clique $K$ on at least three vertices such that

- every vertex in $K$ has at most one neighbor in $N(K)$ and

- every vertex in $N(K)$ has exactly one neighbor in $K$, 
then delete all edges between $K$ and $N(K)$ and decrement $k$ by $q:=|N(K)|$.

Lemma 6.11 Reduction Rule 6.10 is correct and can be exhaustively applied in $O(n m)$ time.

Proof First, enumerate the set of all maximal cliques $K$ on at least three vertices such that every vertex of $K$ has at most one neighbor in $N(K)$. This can be done in $O(m)$ time [29]. Note that every vertex is contained in at most one such clique. Now, by scanning through the adjacency lists of all vertices in an enumerated clique, we can identify a vertex that has more than one neighbor in the clique. If there is such a vertex, then the clique can be discarded. Otherwise, the clique fulfills the conditions of the rule and the rule can be applied. Thus, one application of the rule takes $O(m)$ time. Since the rule decreases the number of vertices in $G$, it can be applied $O(n)$ times.

Since $q \leq|K|$ and $|K| \geq 3$, one can construct $q P_{3}$ s, each containing two vertices from $K$ and one vertex from $N(K)$, such that no two of them share more than one vertex. Thus, at least $q$ edge modifications are needed to destroy all $P_{3}$ s that contain at least one vertex $v \in K$. Deleting all $q$ edges between $K$ and $N(K)$ destroys all $P_{3}$ s that contain at least one vertex of $K$. Moreover, since these edge deletions cut $K$ from the rest of the graph, one can safely combine any optimal solution for $G[V \backslash K]$ with these $q$ edge deletions, which are necessary and sufficient to destroy all $P_{3}$ s that contain at least one vertex of $K$, to obtain an optimal solution that deletes all $q$ edges between $K$ and $N(K)$.

The final rule simply removes isolated clusters from $G$.

Reduction Rule 6.12 If $G$ contains a connected component $K$ that is a clique, then remove $K$ from $G$.

We can now show our improved algorithm for Cluster Editing with $P_{3}$-Packing.

Theorem 6.13 Cluster Editing with $P_{3}$-Packing can be solved in $O\left(4^{\ell} \cdot \ell^{3}+m+\right.$ n) time.

Proof We prove the theorem using a branching algorithm, which applies several branching rules. Herein, we assume that $\mathcal{H}$ contains at least one $P_{3} u v w$. Otherwise, the graph is either $P_{3}$-free (in this case we are done) or we can add a $P_{3}$ to $\mathcal{H}$, which increases $|\mathcal{H}|$ by one and thus reduces the parameter. Furthermore, we assume that Reduction Rules 6.8 to 6.10 and 6.12 do not apply. First, we take care of $P_{3}$ s that do not share an edge with a packing $P_{3}$.

Branching Rule 1: If there is an induced $P_{3}$ that contains at most one vertex of each $P_{3}$ in $\mathcal{H}$, then branch into the three cases to destroy this $P_{3}$.

None of the cases destroys a $P_{3}$ of $\mathcal{H}$. Thus, the parameter is decreased by one in each case; the branching number is 3 .

The three further rules deal with packing $P_{3} \mathrm{~s} u v w$.

Branching Rule 2: If there is a vertex $x$ that is adjacent to $u$ and $w$ but not to $v$, then branch into four cases: delete $\{u, x\}$; delete $\{w, x\}$; add $\{v, x\}$; or delete $\{u, v\}$ and $\{v, w\}$ and add $\{u, w\}$.

In each of the first three branches, $k$ is reduced by one without destroying any $P_{3}$ of $\mathcal{H}$. If none of the first three cases applies, then $u, w$, and $x$ are in the same cluster 
(no edge deletions between $u$ or $w$ and $x$ ) and $v$ is not in this cluster. This makes the three edge modifications in $G[\{u, v, w\}]$ necessary. Thus, $k$ is reduced by three and $|\mathcal{H}|$ is reduced by one in this case. The resulting branching vector is $(1,1,1,2)$, which gives the branching number 3.31.

Branching Rule 3: If there are vertices $x$ and $y$ such that

- $x$ is adjacent to $u$ and $v$, and

- y is adjacent to exactly one vertex of $\{u, v\}$,

then branch into four cases: delete $\{u, x\}$; delete $\{v, x\}$; delete the edge between $y$ and its neighbor in $\{u, v\} ;$ or add the edge between $y$ and its nonneighbor in $\{u, v\}$.

In each case, an edge is modified without destroying any $P_{3}$ of $\mathcal{H}$. If none of the first three cases applies, then $u, v, x$, and $y$ are in the same cluster, which means that the missing edge between $y$ and either $u$ or $v$ has to be added. Since the parameter is reduced by one in each branch, the branching number is 4 .

Branching Rule 4: If there is a vertex $x$ that is adjacent to $v$ and not adjacent to $u$ and $w$, then branch into four cases: delete $\{v, x\}$; add $\{u, x\} ;$ add $\{w, x\}$; or delete $\{u, v\}$ and $\{u, w\}$.

If none of the first three cases applies, then any cluster containing $v$ contains neither $u$ nor $w$, thus the branching is correct. The parameter is reduced by one in each branch, as the last branch destroys a $P_{3}$ of $\mathcal{H}$ but reduces $k$ by two. Thus, the branching number is 4 .

These are the only branching rules that are performed. We now show, by a case distinction, the following: If none of the branching rules and reduction rules applies, then the remaining graph has maximum degree two and we can solve the problem in polynomial time.

Case I: $\mathcal{H}$ contains a $P_{3}$ uvw such that $u$ and $w$ have a common neighbor $x \neq v$. Since Branching Rule 2 does not apply, $x$ is also a neighbor of $v$. Since Branching Rule 3 does not apply, we have that every other vertex $y$ that is adjacent to $u$ is also adjacent to $v$ and vice versa. Similarly, every other vertex $y$ that is adjacent to $w$ is also adjacent to $v$. Thus, $u, v$, and $w$ have the same neighbors in $V \backslash\{u, v, w\}$. This contradicts our assumption that Reduction Rule 6.8 does not apply.

Case II: $\mathcal{H}$ contains a $P_{3}$ uvw such that $v$ has degree at least three. Since Branching Rule 4 does not apply, each vertex $x \in N(v) \backslash\{u, w\}$ is a neighbor of $u$ or $w$ and, since Case I does not apply, it is not a neighbor of both. Moreover, since Branching Rule 3 does not apply, $v$ can have common neighbors with at most one of $u$ and $w$. Thus, without loss of generality, $u$ and $v$ have the same neighborhood in $V \backslash\{u, v, w\}$ and $v$ and $w$ have no common neighbors. This contradicts our assumption that Reduction Rule 6.9 does not apply.

Case III: $\mathcal{H}$ contains a $P_{3} u v w$ such that $u$ has degree at least three. Consider $p$ and $q$ from $N(u) \backslash\{v\}$. Since Case II does not apply, $p$ and $q$ are not middle vertices of a $P_{3}$ in $\mathcal{H}$. Moreover, since Case I does not apply, $p$ and $q$ are not from the same $P_{3}$ of $\mathcal{H}$. Consequently, if $G[\{u, p, q\}]$ is a $P_{3}$, then Branching Rule 1 applies. This implies that $G[N[u] \backslash\{v\}]$ is a clique $K$ of size at least three. We now show the following claim, which contradicts our assumption that Reduction Rule 6.10 does not apply.

Claim: Each vertex of $K$ has at most one neighbor in $V \backslash K$ and every vertex in $N(K)$ has at most one neighbor in $K$. 
First, observe that no vertices from $K$ are middle vertices of a $P_{3}$ in $\mathcal{H}$. Thus, $K$ contains at most one vertex $x$ from each $P_{3}$ of $\mathcal{H}$ and this vertex $x$ is not a middle vertex. For each such $x \in K$ from a packing $P_{3} x y z$, the same conditions apply as to $u$, thus $G[N[x] \backslash\{y\}]$ is a clique $K^{\prime}$. This implies $K=K^{\prime}$ : Since $G[N[u] \backslash\{v\}]$ is a clique, $x$ is adjacent to every vertex in $N[u] \backslash\{v\}$ and since $G[N[x] \backslash\{y\}]$ is a clique, $u$ is adjacent to every vertex in $N[x] \backslash\{y\}$. Summarizing, each vertex from $K$ that is in a $P_{3}$ of $\mathcal{H}$ has exactly one neighbor outside of $K$, this neighbor is a middle vertex of the packing $P_{3}$ containing $x$. These middle vertices have only one neighbor in $K$ since they have degree two and $K$ contains only one vertex from each packing $P_{3}$.

Now let $x$ denote a vertex of $K$ that is not contained in any $P_{3}$ of $\mathcal{H}$. We show that $N[x]=K$, which implies the claim. Since the middle vertices of each $P_{3}$ of $\mathcal{H}$ have no neighbors outside of this $P_{3}$ and since Branching Rule 2 does not apply, we have that $x$ is adjacent to at most one vertex of each packing $P_{3}$. Thus, $G[N[x]]$ is a clique $K^{\prime}$ as otherwise, Branching Rule 1 applies. Again, $K^{\prime}=K$ as $G[N[u] \backslash\{v\}]$ being a clique implies that $x$ is adjacent to every vertex in $N[u] \backslash\{v\}$ and $G[N[x]]$ being a clique implies that $u$ is adjacent to every vertex in $N[x]$.

Case IV: otherwise. We show that every vertex has degree at most two. This is true for the middle vertices of $P_{3} \mathrm{~s}$ in $\mathcal{H}$ as Case II does not apply. This also holds for the endpoints of $P_{3} \mathrm{~s}$ in $\mathcal{H}$ as Case III does not apply. We now argue that every other vertex $x$ cannot have two neighbors.

The vertex $x$ has at least one neighbor $u$ from some $P_{3} u v w$ of $\mathcal{H}: x$ is contained in at least one $P_{3}$ because the instance is reduced with respect to Reduction Rule 6.12 and this $P_{3}$ contains at least two vertices of some $P_{3}$ of the packing because Branching Rule 1 does not apply. If $x$ has a further neighbor $y$, then $y \neq v$ (since Case II does not apply) and $v \neq w$ since (Case I) does not apply. Consequently, $u, x$, and $y$ form a triangle (since Branching Rule 1 does not apply). Thus, $u$ has two neighbors outside of his $P_{3}$ of $\mathcal{H}$, which means that Case III applies.

Thus, $G$ has maximum degree two and does not contain isolated triangles because the instance is reduced with respect to Reduction Rule 6.12. In this case, an optimal solution can be obtained by computing a maximum matching $M$ and then deleting all edges of $G$ that are not in $M$.

Altogether, the above considerations imply a search tree algorithm with search tree size $O\left(4^{\ell}\right)$. After an initial kernelization, which, due to Corollary 6.7, runs in $O(m+n)$ time for $t=1$, the instance has $O(\ell)$ vertices. Thus, the steps at each search tree node including the reduction rules can be performed in $O\left(\ell^{3}\right)$ time.

\section{Hardness Results for Edge Deletion and Vertex Deletion Problems}

In this section, we show edge modification problems and vertex deletion problems that are NP-hard even for small forbidden induced subgraphs and if $\ell=k-|\mathcal{H}|$, where $\mathcal{H}$ is a vertex-disjoint packing of forbidden induced subgraphs. We also show that algorithms for VerTEX COVER parameterized above lower bounds do not generalize to $d$-Hitting SET — the natural generalization of VeRTex Cover to hypergraphs. 
Theorem 7.1 For every fixed $q \geq 6, K_{q}$-FREe Deletion with $K_{q}$-Packing is NP-hard for $\ell=0$.

We prove Theorem 7.1 by giving a reduction from 3-SAT (Problem 4.10).

Construction 7.2 Let $\phi$ be a Boolean formula with variables $x_{1}, \ldots, x_{n}$ and clauses $C_{1}$, $\ldots, C_{m}$. We assume that each clause $C_{j}$ contains exactly three pairwise distinct variables. We create a graph $G$ and a vertex-disjoint $K_{q}$-packing $\mathcal{H}$ as follows.

For each variable $x_{i}$, add a clique $X_{i}$ on $q$ vertices to $G$ that has two distinguished disjoint edges $x_{i}^{\mathrm{F}}$ and $x_{i}^{\mathrm{T}}$. For each clause $C_{j}=\left(l_{1} \wedge l_{2} \wedge l_{3}\right)$ with literals $l_{1}, l_{2}$, and $l_{3}$, add a clique $Y_{j}$ on $q$ vertices to $G$ that has three distinguished and pairwise disjoint edges $e_{l_{1}}, e_{l_{2}}$, and $e_{l_{3}}$ (which exist since $q \geq 6$ ). Finally, if $l_{t}=x_{i}$, then identify the edge $e_{l}$ with $x_{i}^{\mathrm{T}}$ and if $l_{t}=\neg x_{i}$, then identify the edge $e_{l_{t}}$ with $x_{i}^{\mathrm{F}}$. The packing $\mathcal{H}$ consists of all $X_{i}$ introduced for the variables $x_{i}$ of $\phi$.

Lemma 7.3 Let $G$ be the graph output by Construction 7.2 and let $H$ be an induced $K_{q}$ in $G$. Then, $H$ is either one of the $X_{i}$ or one of the $Y_{j}$.

Proof First, note that the $X_{i}$ are pairwise vertex-disjoint since Construction 7.2 only identifies edges of $Y_{j}$ s with edges of $X_{i}$ s and no edge in any $Y_{j}$ is identified with edges in different $X_{i}$. For any $X_{i}$ and $Y_{j}$, the vertices in $V\left(X_{i}\right) \backslash V\left(Y_{j}\right)$ are nonadjacent to those in $V\left(Y_{j}\right) \backslash V\left(X_{i}\right)$. Similarly, for $Y_{i}$ and $Y_{j}$, the vertices in $V\left(Y_{i}\right) \backslash V\left(Y_{j}\right)$ are nonadjacent to those in $V\left(Y_{j}\right) \backslash V\left(Y_{i}\right)$ for $i \neq j$. Thus, every clique in $G$ is entirely contained in one of the $X_{i}$ or $Y_{j}$.

Lemma 7.3 allows us to prove Theorem 7.1.

Proof (of Theorem 7.1) We show that $\phi$ is satisfiable if and only if $G$ can be made $K_{q}$-free by $k=|\mathcal{H}|$ edge deletions (that is, $\ell=0$ ).

First, assume that there is an assignment that satisfies $\phi$. We construct a $K_{q}$-free deletion set $S$ for $G$ as follows: if the variable $x_{i}$ is set to true, then put $x_{i}^{\mathrm{T}}$ into $S$. If the variable $x_{i}$ is set to false, then add $x_{i}^{\mathrm{F}}$ to $S$. Thus, for each $X_{i}$, we add exactly one edge to $S$. Since $\mathcal{H}$ consists of the $X_{i}$, we have $|S|=|\mathcal{H}|$. Moreover, since each clause $C_{j}$ contains a true literal, at least one edge of each $Y_{j}$ is contained in $S$. Thus, $G \backslash S$ is $K_{q}$-free, since, by Lemma 7.3, the only $K_{q}$ s in $G$ are the $X_{i}$ and $Y_{j}$ and, for each of them, $S$ contains at least one edge.

Now, assume that $G$ can be made $K_{q}$-free by deleting a set $S$ of $|\mathcal{H}|$ edges. Then, $S$ deletes exactly one edge of each $X_{i}$ and at least one edge of each $Y_{j}$. We can assume without loss of generality that $S$ contains either the edge $x_{i}^{\mathrm{T}}$ or $x_{i}^{\mathrm{F}}$ for each $X_{i}$ since deleting one of these edges instead of another edge in $X_{i}$ always yields a solution by Construction 7.2. Thus, the deletion set $S$ corresponds to a satisfying assignment for $\phi$. 


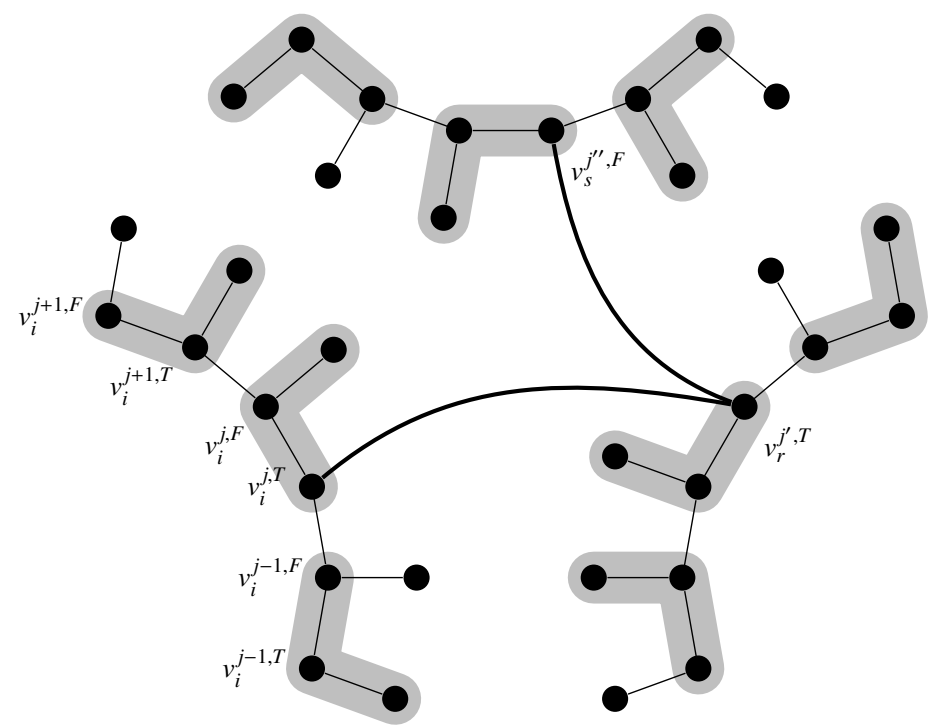

Fig. 7.1 An illustration of Construction 7.6 for $q=3$. The figure shows parts of the variable cycles for three variables $x_{i}, x_{r}, x_{s}$ that occur in the clause $C_{t}=\left(x_{i} \vee x_{r} \vee \neg x_{s}\right)$. The packing $P_{3} \mathrm{~s}$ are highlighted by a gray background.

\subsection{Hard vertex deletion problems}

In this section, we show NP-hardness of the problem of destroying all induced paths $P_{q}$ on $q \geq 3$ vertices by at most $|\mathcal{H}|$ vertex deletions if a packing $\mathcal{H}$ vertex-disjoint induced $P_{q}$ s in the input graph $G$ is provided as input.

\section{Problem 7.4 ( $\boldsymbol{P}_{q}$-Free Vertex Deletion with $\boldsymbol{P}_{q}$-Packing $)$}

Input: A graph $G=(V, E)$, a vertex-disjoint packing $\mathcal{H}$ of induced $P_{q}$ s, and a natural number $k$

Question: Is there a vertex set $S \subseteq V$ of size at most $k$ such that $G[V \backslash S]$ does not contain $P_{q}$ as induced subgraph?

Theorem 7.5 For every fixed $q \geq 3, P_{q}$-Free $V_{\text {ertex }}$ Deletion with $P_{q}$-Packing is $N P$-hard even if $\ell=0$.

The reduction is from $q$-SAT:

Construction 7.6 Let $\phi$ be a Boolean formula with variables $x_{1}, \ldots, x_{n}$ and clauses $C_{1}$, $\ldots, C_{m}$. We assume that each clause $C_{j}$ contains exactly $q$ pairwise distinct variables. We construct a graph $G$ and a maximal vertex-disjoint packing $\mathcal{H}$ of $P_{q}$ s as follows; an illustration of the construction is given in Figure 7.1.

First, we introduce variable gadgets, which will ensure that a solution to $P_{q}$-FREE Vertex Deletion with $P_{q}$-Packing corresponds to an assignment of $\phi$. In the following, let $\alpha(i)$ denote the number of occurrences of $x_{i}$ and $\neg x_{i}$ in clauses of $\phi$. For each variable $x_{i}$, add $4 \alpha(i)$ vertices: $v_{i}^{j, \mathrm{~T}}$ and $v_{i}^{j, \mathrm{~F}}$, where $1 \leq j \leq 2 \alpha(i)$. Call $v_{i}^{j, \mathrm{~T}}$ a true vertex 
and $v_{i}^{j, \mathrm{~F}}$ a false vertex. Create an induced cycle on the true and false vertices by adding the edge set

$$
E_{i}:=\left\{\left\{v_{i}^{j, \mathrm{~T}}, v_{i}^{j, \mathrm{~F}}\right\} \mid 1 \leq j \leq 2 \alpha(i)\right\} \cup\left\{\left\{v_{i}^{j, \mathrm{~F}}, v_{i}^{j+1, \mathrm{~T}}\right\} \mid 1 \leq j<2 \alpha(i)\right\} \cup\left\{\left\{v_{i}^{2 \alpha(i), \mathrm{F}}, v_{i}^{1, \mathrm{~T}}\right\}\right\} .
$$

Call this cycle the variable cycle of $x_{i}$.

Then, for each even $j$, attach to $v_{i}^{j, T}$ an induced $P_{q-2}$, that is, make one of its degree-one vertices adjacent to $v_{i}^{j, \mathrm{~T}}$. Then, again for each even $j$, attach to $v_{i}^{j, \mathrm{~F}}$ an induced $P_{q-2}$ in the same fashion. These paths are called the attachment paths of the $j$ th segment of the variable cycle of $x_{i}$.

Now, for each variable $x_{i}$, assign to each clause $C_{t}$ containing $x_{i}$ or $\neg x_{i}$ a unique number $p \in\{1, \ldots, \alpha(i)\}$. Consider the number $j=2 p-1$. We will use vertex $v_{i}^{j, \mathrm{~T}}$ or $v_{i}^{j, \mathrm{~F}}$ to build the clause gadget for clause $C_{t}$. If $C_{t}$ contains the literal $x_{i}$, then attach an induced $P_{q-2}$ to $v_{i}^{j, \mathrm{~F}}$. Otherwise, attach an induced $P_{q-2}$ to $v_{i}^{j, \mathrm{~T}}$. As above, call the path the attachment path of the $j$ th segment of the cycle. Now, let $\gamma_{i}^{t}:=v_{i}^{j, \mathrm{~T}}$ if $C_{t}$ contains $x_{i}$, and let $\gamma_{i}^{t}:=v_{i}^{j, \mathrm{~F}}$ if $C_{t}$ contains $\neg x_{i}$. Call these vertices the literal vertices of clause $C_{t}$, denoted $\Pi_{t}$. The construction of $G$ is completed as follows. For each $\Pi_{t}$ add an arbitrary set of edges to $G$ such that $G\left[\Pi_{t}\right]$ is an induced $P_{q}$. The $P_{q}$-packing $\mathcal{H}$ contains one (arbitrary) attachment path plus the two segment vertices from each segment of each variable cycle.

Proof (of Theorem 7.5) Let $G$ be the graph output by Construction 7.6 and let $\mathcal{H}$ be the $P_{q}$-packing. We show that $\phi$ has a satisfying assignment if and only if $G$ can be made $P_{q}$-free by exactly $|\mathcal{H}|$ vertex deletions (that is, $\ell=0$ ).

Assume that $\phi$ has a satisfying assignment. For each true variable $x_{i}$ in this assignment, delete all true vertices in its variable gadget, that is, $v_{i}^{j, \mathrm{~T}}$ for $1 \leq j \leq 2 \alpha(i)$. For each false variable $x_{i}$ in this assignment, delete all false vertices in its variable gadget, that is, $v_{i}^{j, \mathrm{~F}}$ for $1 \leq j \leq 2 \alpha(i)$. Denote this vertex set by $S$ and observe that $|S|=|\mathcal{H}|$. Moreover, observe that each vertex on the variable cycle for $x_{i}$ is either deleted or both of its neighbors on the cycle are deleted. Every $P_{q}$ in $G$ contains at least one vertex from a variable cycle as the attachment paths are too short to induce $P_{q} \mathrm{~s}$. Thus, to show $P_{q}$-freeness of $G[V \backslash S]$ it is sufficient to show that no vertex from a variable cycle is in a $P_{q}$.

Consider an undeleted vertex in the variable cycle for $x_{i}$. Assume, without loss of generality, that this is a true vertex $v_{i}^{j, \mathrm{~T}}$. If $j$ is even, then $v_{i}^{j, \mathrm{~T}}$ is not in a $P_{q}$ as its neighbors on the cycle are deleted and its only other neighbor is in an attachment path. If $j$ is odd and the clause $C_{t}$ corresponding to the $j$ th segment of the cycle contains $\neg x_{i}$, then the only neighbor of $v_{i}^{j, \mathrm{~T}}$ in $G[V \backslash S]$ is in an attachment path. It remains to show that $v_{i}^{j, \mathrm{~T}}$ is not in a $P_{q}$ if $C_{t}$ contains $x_{i}$. The only neighbors of $v_{i}^{j, \mathrm{~T}}$ in $G[V \backslash S]$ are in $\Pi_{t}$. Observe that $G\left[\Pi_{t}\right]$ is an induced $P_{q}$ and that, in $G[V \backslash S]$, every vertex on this path is deleted or its neighbors in $V \backslash \Pi_{t}$ are deleted. Hence, the connected component of $G[V \backslash S]$ containing $v_{i}^{j, T}$ is an induced subgraph of $G\left[\Pi_{t}\right]$. Since the assignment is satisfying, at least one vertex of $\Pi_{t}$ is deleted. Thus, this connected component has at most $q-1$ vertices and does not contain a $P_{q}$.

Conversely, let $S \subseteq V$ be a size- $|\mathcal{H}|$ vertex set such that $G[V \backslash S]$ is $P_{q}$-free. First, observe that, without loss of generality, for each variable cycle either all true or all 
false vertices are deleted: No vertex in an attachment path $P$ is deleted since it is always as good to delete the vertex in the variable cycle that has a neighbor in $P$. Hence, at least one vertex of each segment is deleted since, otherwise, one of the $P_{q}$ 's in $\mathcal{H}$ is not destroyed. This already requires $|\mathcal{H}|$ vertex deletions and thus exactly one vertex for each segment of each variable cycle is deleted. Finally, by construction, every adjacent pair of vertices in the variable cycle forms a $P_{q}$ with some attachment path. Therefore, one of the two vertices is deleted, which implies that either every even or every odd vertex of the cycle is deleted.

Hence, the vertex deletions in the variable cycle define a truth assignment $\beta$ to $x_{1}, \ldots, x_{n}$ : If all true vertices of the variable cycle of $x_{i}$ are deleted, then set $\beta\left(x_{i}\right):=$ true; otherwise, set $\beta\left(x_{i}\right):=$ false. This assignment is satisfying: Since $G[V \backslash S]$ is $P_{q}$-free, for each clause $C_{t}$, at least one vertex $\gamma_{i}^{t}$ of $\Pi_{t}$ is deleted. Without loss of generality, let $\gamma_{i}^{t}=v_{i}^{j, \mathrm{~T}}$, that is, $C_{t}$ contains the literal $x_{i}$. Then, $\beta\left(x_{i}\right)=$ true and thus $\beta$ satisfies clause $C_{t}$.

Theorem 7.5 easily transfers to a hardness result for the generalization of VERTEX Cover to $d$-uniform hypergraphs:

\section{Problem 7.7 ( $d$-Uniform Hitting Set with Packing)}

Input: A hypergraph $H=(V, E)$ with $|e|=d$ for all $e \in E$, a set $\mathcal{H} \subseteq E$ of pairwise vertex-disjoint hyperedges, and an integer $k$.

Question: Is there a vertex set $V^{\prime} \subseteq V$ of size at most $k$ such that $\forall e \in E: V^{\prime} \cap e \neq \emptyset$ ?

An instance $(G, \mathcal{H}, k)$ of $P_{q}$-Free Vertex Deletion with $P_{q}$-Packing can easily be transformed into an equivalent instance $(H, \mathcal{H}, k)$ of $q$-Uniform Hitting Set with PACKING by taking the hypergraph $H$ on the same vertex set as $G$ having a hyperedge $e$ if and only if $G[e]$ is a $P_{q}$. The packing $\mathcal{H}$ and $k$ stay unchanged, and so does $\ell$. Thus, we obtain the following result:

Corollary 7.8 For every $d \geq 3, d$-Uniform Hitting Set with Packing is NP-hard even if $\ell=0$.

Corollary 7.8 shows that the known above-guarantee fixed-parameter algorithms for Vertex Cover $[13,22,36,43]$ do not generalize to $d$-Uniform Hitting SET.

\section{Conclusion}

It is open to extend our framework to further problems. The most natural candidates appear to be problems where the forbidden induced subgraph has four vertices. Examples are Cograph Editing [35] which is the problem of destroying all induced $P_{4}$ s, $K_{4}$-FreE Editing, Claw-free Editing, and Diamond-free Deletion [18, 44]. Another direction could be to investigate edge completion problems that allow for subexponential-time algorithms [15]. In the case of vertex-deletion problems, Triangle Vertex Deletion appears to be the most natural open case. Furthermore, it would be nice to obtain more general theorems separating the tractable from the hard cases for this parameterization. For Cluster Editing and Triangle Deletion improved running times are desirable. 
Maybe more importantly, it is open to determine the complexity of CLUSTER EDITING and Feedback Arc Set in Tournaments parameterized above the size of edge-disjoint packings of forbidden induced subgraphs. Finally, our framework offers an interesting tradeoff between running time and power of generic data reduction rules. Exploring such tradeoffs seems to be a rewarding topic for the future. The generic rules presented in this work can be easily implemented, which asks for subsequent experiments to evaluate their effectiveness.

\section{References}

1. Alon N (2006) Ranking tournaments. SIAM Journal on Discrete Mathematics 20(1):137-142

2. Alon N, Lokshtanov D, Saurabh S (2009) Fast FAST. In: Proceedings of the 36th International Colloquium on Automata, Languages, and Programming (ICALP '09), Springer, Lecture Notes in Computer Science, vol 5555, pp 49-58

3. Aravind NR, Sandeep RB, Sivadasan N (2016) Parameterized lower bounds and dichotomy results for the NP-completeness of $H$-free edge modification problems. In: Proceedings of the 12th Latin American Symposium on Theoretical Informatics, (LATIN'16), vol 9644, pp 82-95

4. Bessy S, Fomin FV, Gaspers S, Paul C, Perez A, Saurabh S, Thomassé S (2011) Kernels for feedback arc set in tournaments. Journal of Computer and System Sciences 77(6): 1071-1078

5. van Bevern R (2014) Towards optimal and expressive kernelization for $d$-Hitting Set. Algorithmica 70(1):129-147

6. van Bevern R, Froese V, Komusiewicz C (2016) Parameterizing edge modification problems above lower bounds. In: Proceedings of the 11th International Computer Science Symposium in Russia (CSR'16), Springer, Lecture Notes in Computer Science, vol 9691, pp 57-72

7. Böcker S (2012) A golden ratio parameterized algorithm for cluster editing. Journal of Discrete Algorithms 16:79-89

8. Böcker S, Briesemeister S, Bui QBA, Truß A (2009) Going weighted: Parameterized algorithms for cluster editing. Theoretical Computer Science 410(52):54675480

9. Brügmann D, Komusiewicz C, Moser H (2009) On generating triangle-free graphs. In: Proceedings of the DIMAP Workshop on Algorithmic Graph Theory (AGT '09), Elsevier, ENDM, pp 51-58

10. Cai L (1996) Fixed-parameter tractability of graph modification problems for hereditary properties. Information Processing Letters 58(4):171-176

11. Cai L, Cai Y (2015) Incompressibility of $H$-free edge modification problems. Algorithmica 71(3):731-757

12. Chen J, Meng J (2012) A $2 k$ kernel for the cluster editing problem. Journal of Computer and System Sciences 78(1):211-220

13. Cygan M, Pilipczuk M, Pilipczuk M, Wojtaszczyk JO (2013) On multiway cut parameterized above lower bounds. ACM Transactions on Computation Theory $5(1): 3$ 
14. Dom M, Guo J, Hüffner F, Niedermeier R, TrußA (2006) Fixed-parameter tractability results for feedback set problems in tournaments. In: Proceedings of the 6th International Conference on Algorithms and Complexity (CIAC '06), Springer, Lecture Notes in Computer Science, vol 3998, pp 320-331

15. Drange PG, Fomin FV, Pilipczuk M, Villanger Y (2015) Exploring the subexponential complexity of completion problems. ACM Transactions on Computation Theory 7(4): 14

16. Feige U (2009) Faster FAST (feedback arc set in tournaments). CoRR abs/0911.5094, URL http: //arxiv .org/abs/0911. 5094

17. Fellows MR (2003) Blow-ups, win/win's, and crown rules: Some new directions in FPT. In: Proceedings of the 29th International Workshop on Graph-Theoretic Concepts in Computer Science (WG '03), Lecture Notes in Computer Science, vol 2880, pp 1-12

18. Fellows MR, Guo J, Komusiewicz C, Niedermeier R, Uhlmann J (2011) Graphbased data clustering with overlaps. Discrete Optimization 8(1):2-17

19. Fomin FV, Kratsch D (2010) Exact Exponential Algorithms. Texts in Theoretical Computer Science. An EATCS Series, Springer

20. Fomin FV, Pilipczuk M (2013) Subexponential parameterized algorithm for computing the cutwidth of a semi-complete digraph. In: Proceedings of the 21st Annual European Symposium (ESA '13), Springer, Lecture Notes in Computer Science, vol 8125, pp 505-516

21. Fomin FV, Kratsch S, Pilipczuk M, Pilipczuk M, Villanger Y (2011) Subexponential fixed-parameter tractability of cluster editing. CoRR abs/1112.4419, URL http: //arxiv.org/abs/1112.4419

22. Garg S, Philip G (2016) Raising the bar for vertex cover: Fixed-parameter tractability above a higher guarantee. In: Proceedings of the 27th Annual ACM-SIAM Symposium on Discrete Algorithms (SODA '16), SIAM, pp 1152-1166

23. Gramm J, Guo J, Hüffner F, Niedermeier R (2004) Automated generation of search tree algorithms for hard graph modification problems. Algorithmica 39(4):321347

24. Guillemot S, Havet F, Paul C, Perez A (2013) On the (non-)existence of polynomial kernels for $P_{\ell}$-free edge modification problems. Algorithmica 65(4):900-926

25. Guo J (2009) A more effective linear kernelization for cluster editing. Theoretical Computer Science 410(8-10):718-726

26. Hartung S, Hoos HH (2015) Programming by optimisation meets parameterised algorithmics: A case study for cluster editing. In: Proceedings of the 9th International Conference on Learning and Intelligent Optimization (LION '15), Springer, Lecture Notes in Computer Science, vol 8994, pp 43-58

27. Karpinski M, Schudy W (2010) Faster algorithms for feedback arc set tournament, kemeny rank aggregation and betweenness tournament. In: Proceedings of the 21st International Symposium on Algorithms and Computation (ISAAC '10), Springer, Lecture Notes in Computer Science, vol 6506, pp 3-14

28. Komusiewicz C, Uhlmann J (2012) Cluster editing with locally bounded modifications. Discrete Applied Mathematics 160(15):2259-2270

29. Komusiewicz C, Hüffner F, Moser H, Niedermeier R (2009) Isolation concepts for efficiently enumerating dense subgraphs. Theoretical Computer Science 410(38- 
40):3640-3654

30. Kratsch S (2012) Polynomial kernelizations for $\mathrm{MIN} \mathrm{F}^{+} \Pi_{1}$ and MAX NP. Algorithmica 63(1-2):532-550

31. Kratsch S (2014) Recent developments in kernelization: A survey. Bulletin of the EATCS 113

32. Kratsch S, Wahlström M (2013) Two edge modification problems without polynomial kernels. Discrete Optimization 10(3):193-199

33. Kř́ivánek M, Morávek J (1986) NP-hard problems in hierarchical-tree clustering. Acta Informatica 23(3):311-323

34. Lewis JM, Yannakakis M (1980) The node-deletion problem for hereditary properties is NP-complete. Journal of Computer and System Sciences 20(2):219-230

35. Liu Y, Wang J, Guo J, Chen J (2012) Complexity and parameterized algorithms for Cograph Editing. Theoretical Computer Science 461:45-54

36. Lokshtanov D, Narayanaswamy NS, Raman V, Ramanujan MS, Saurabh S (2014) Faster parameterized algorithms using linear programming. ACM Transactions on Algorithms 11(2):15:1-15:31

37. Mahajan M, Raman V (1999) Parameterizing above guaranteed values: MaxSat and MaxCut. Journal of Algorithms 31(2):335-354

38. Moser H, Niedermeier R, Sorge M (2012) Exact combinatorial algorithms and experiments for finding maximum $k$-plexes. Journal of Combinatorial Optimization 24(3):347-373

39. Niedermeier R (2006) Invitation to Fixed-Parameter Algorithms. Oxford University Press

40. Paul C, Perez A, Thomassé S (2016) Linear kernel for rooted triplet inconsistency and other problems based on conflict packing technique. Journal of Computer and System Sciences 82(2):366-379

41. Protti F, da Silva MD, Szwarcfiter JL (2009) Applying modular decomposition to parameterized cluster editing problems. Theory of Computing Systems 44(1):91104

42. Raman V, Saurabh S (2006) Parameterized algorithms for feedback set problems and their duals in tournaments. Theoretical Computer Science 351(3):446-458

43. Razgon I, O'Sullivan B (2009) Almost 2-sat is fixed-parameter tractable. Journal of Computer and System Sciences 75(8):435-450

44. Sandeep RB, Sivadasan N (2015) Parameterized lower bound and improved kernel for diamond-free edge deletion. In: Proceedings of the 10th International Symposium on Parameterized and Exact Computation (IPEC '15), Schloss Dagstuhl Leibniz-Zentrum fuer Informatik, LIPIcs, vol 43, pp 365-376

45. Shamir R, Sharan R, Tsur D (2004) Cluster graph modification problems. Discrete Applied Mathematics 144(1-2):173-182

46. Wahlström M (2007) Algorithms, measures and upper bounds for satisfiability and related problems. $\mathrm{PhD}$ thesis, Linköpings universitet

47. Yannakakis M (1981) Edge-deletion problems. SIAM Journal on Computing 10(2):297-309 